\title{
THEORY AND MEASUREMENT OF VISUAL MECHANISMS
}

\section{On Flicker wIth SUbdivided FIElds}

BY W. J. CROZIER AND ERNST WOLF

(From the Biological Laboratories, Harvard University, Cambridge)

(Received for publication, December 17, 1943)

I

When visual flicker is produced by passing vertical dark bars across an illuminated field ${ }^{1}$ with fixed inclined opaque stripes on it, certain pronounced changes are induced in the properties of the flicker contour. ${ }^{2,3}$

Ordinarily, with image fields not subdivided, and regardless of whether flicker is produced by sectoring the light beam at a focus ${ }^{4}$ or by the striped cylinder technic, the dependence of the contour for ficker recognition on the light-time fraction $t_{L}$ in the flash cycle is direct and simple. ${ }^{5}$ The maximum flash frequency $F$ to which the $F-\log I$ curve asymptotically rises declines rectilinearly with increase of $t_{L}$; its abscissa of inflection (photopic "cone" segment) increases rectilinearly with $t_{L}$; and the third parameter of the probability summation ${ }^{6}$ describing the simplex $F-\log I$ curve, namely the standard deviation $\sigma_{\log I}^{\prime}$ of its first derivative in $\log I$, with $F_{\max }$. put $=100$, is found not to change at all when $t_{L}$ is altered. (In certain cases, with most arthropods, ${ }^{7}$ slight changes in the shape of the lower part of the curve arise as a consequence of the convexity of the surface of the eye, but this does not affect the essential generality of the previous statement.) Fundamentally, the behavior of the scotopic "rod" part of duplex $F-\log I$ contours is the same, but this may be obscured by the integrated overlapping of the "rod" and "cone" populations of neural effects. ${ }^{8}$

With an obliquely barred field, however, and flicker produced by evenly

1 Wolf, E., and Zerrahn-Wolf, G., 1935-36, J. Gen. Physiol., 19, 495. Crozier, W. J., Wolf, E., and Zerrahn-Wolf, G., 1936-37, J. Gen. Physiol., 20, 211; 1937-38, 21, 203 , etc.

2 1941-42, J. Gen. Physiol., 25, 369.

3 1943-44, J. Gen. Physiol., 27, 287.

${ }^{4}$ 1940-41, J. Gen. Physiol., 24, 505, 635; 1941-42, 25, 89, 293; 1943-44, 27, 119.

- 5 1937-38, J. Gen. Physiol., 21, 313, 463; Proc. Nat. Acad. Sc., 1940, 26, 60; J. Gen. Physiol., 1939-40, 23, 531.

6 1938-39, J. Gen. Physiol., 22, 311, 451; 1940-41, 24, 625; 1941-42, 25, 369; $1943-$ 44, 27, 119.

7 1937-38, J. Gen. Physiol., 21, 463.

8 1937-38, J. Gen. Physiol., 21, 313; 1940-41, 24, 635.

401 
spaced vertical bars moving across it, the shape constant $\sigma_{\log I}^{\prime}$ is sharply decreased above $t_{L}=0.50$, so that the contour is then much steeper; $\tau^{\prime}$ does not increase as much as with a plain field, and neither $\tau^{\prime}$ nor $F_{\max }$. is a simple function of $t_{L}$. These effects cause the curves for large values of $t_{L}$ to cut across those for the smaller values, instead of being evenly and symmetrically spaced as with the plain field.

This latter picture is the one obtained for the sets of $F-\log I$ contours at different values of $t_{L}$ secured with birds, ${ }^{3,9}$ by the revolving stripe method. The birds used possess large, well developed pectens. The theory ${ }^{3}$ is that the moving contact of bar images with the serrated shadow of the pecten causes the natural occurrence of the phenomenon experimentally induced in man by the use of the obliquely barred field.

Partly as a test of this conception, partly for other reasons, we have examined the results to be obtained when transilluminated parts of stationary barred patterns of different sorts are caused to flicker simultaneously by sectoring the light at a focus. The point then is that, a standing pattern being intermittently illuminated, one can inquire whether subdivision of the flickered field into several parts will of itself introduce modification of the properties of the set of flicker contours when $t_{L}$ is varied, and whether such modification will be similar to that involved in the "pecten effect." From another standpoint, it is to be noted that we can also examine by this means the problem of neural integration in visual excitation. ${ }^{10}$ The relation between $F$ and $\log I$ critical for flicker, although simultaneously apparent in the several parts of a subdivided field, is not the same as when one of its parts is tested separately or when a simple field of the same general form and the same total illuminated area is flickered.

We are more immediately concerned with the demonstration that the "pecten effect" is only in part reproduced in the changes actually effected in the properties of the $F-\log I$ contour by the "stationary flickering" of subdivided fields. The character of the changes found substantiates the importance of the "sliding contact" of stationary and moving dark images for the production of enhanced flicker acuity by the "pecten effect."

The results here given also extend the basis for an understanding of the manner in which "rod" and "cone" effects are integrated to produce the $F-$ $\log I$ contour in the region of their overlapping. They likewise bear directly upon the curious properties of "visual acuity" with interrupted light, which we discuss in a following paper. And, in a more general connection, the data here cited complete the proof that three independently modifiable parameters $\left(F_{\max .}, \tau^{\prime}, \sigma_{\log I}^{\prime}\right)$ are involved in the nature of the flicker contour.

${ }^{9}$ 1943-44, J. Gen. Physiol., 27, 315.

${ }^{10} \mathrm{Cf}$. 1940-41, J. Gen. Physiol., 24, 505; 1941-42, 25, 369; and a following paper. 
II

The general procedure followed in these experiments, the instrumentation, and the methods of calculation, have been described previously. ${ }^{11}$ The four fields used for the present discussion are: (i) a square subtending at the retina $3^{\circ}$ on a side, centered $6^{\circ}$ horizontally on the temporal aspect of the left retina; (ii) a square, subtending $3^{\circ}$ on a side, with horizontal and vertical subdivision into four equal squares (Fig. 1), the opaque dividing stripes being $0.3^{\circ}$ broad; centered as for (i). The crossed bars were formed of pieces of wristwatch hair-spring mounted in the jaws of the spectroscopic slit in one arm of our discriminometer. ${ }^{12}$ The total illuminated area with (i) and (ii) was taken to be nearly enough the same, despite scattered light on the crossbars. (iii) A square subtending $10^{\circ}$ on a side, centered at the fovea, with three opaque vertical bars each $1.43^{\circ}$ wide, thus giving four vertical illumined spaces of that width, equally separated; and (iv) a square $10^{\circ}$ (really $9.91^{\circ}$ ) on a side with six opaque vertical bars and seven illuminated bars all of $0.77^{\circ}$ width. Thus the bars in (iv) were of about one-half the width of those in (iii), and the total illuminated area in (iv) was a little less (52.9 square degrees as compared with 57.2 square degrees). For certain tests field (iv) was rotated $90^{\circ}$ so that the stripes were horizontal; we may speak of this as field (v). The opaque bars were produced by hard photographic reduction of carefully made contact prints of Levy plates, mounted in the spectroscopic slits of the discriminometer.

The observations were made monocularly (left eye). Tungsten white light was used with (ii), and the white and a red and a blue filtered from it with the other fields.

III

The data for fields (i) and (ii) are given in Tables I and II. In Figs. 1 and 2 it is seen that the nature of the shift in contours with change of light-time fraction $t_{L}$ is of the kind already found for plain fields. ${ }^{11}$ In the subdivided field (ii) there is no change of the slope constant at $t_{L}=0.90$, nor any change in the type of relation of $\tau^{\prime}$ to $t_{L}$; and in this sense there is no pronounced "pecten effect." 3 But the picture is nevertheless changed considerably when the $3^{\circ}$ square is subdivided into four parts. The "rod" segment is then greatly enlarged, the "cone" segment becomes much steeper, and its midpoint is moved to a higher flash intensity, the interrelation of these two phenomena we shall consider shortly. The slight increase in over-all illuminated area in (ii), by scattered light on the crossbar images, cannot possibly account for them. ${ }^{13}$ The steepening of the "cone" segment is a prominent feature of the "pecten effect," but when this effect occurs it is seen only with longer light-times in the flash cycle $;^{3,9}$ here it is independent of $t_{L}$. It is perfectly clear, as already emphasized elsewhere, ${ }^{10}$ that pronounced changes of the shape constants of the flicker contours make futile any attempt to deduce from such constants alone

11 1940-41, J. Gen. Physiol., 24, 505, 635; 1941-42, 25, 89, 293.

12 1938-39, J. Gen. Physiol., 22, 341.

${ }^{13} \mathrm{Cf}$. following paper. 
TABLE I

Data for flicker recognition contours with a $3^{\circ}$ square image centered $6^{\circ}$ on the temporal side of the fovea (W. J. C., monocular observations with left eye); white light; flash intensities $I$ in millilamberts; " $t_{L}$ " $=t_{L} /\left(t_{L}+t_{D}\right) ; n=10$ for each point.

\begin{tabular}{|c|c|c|c|c|c|c|}
\hline$\underset{\text { per sec. }}{F}$ & \multicolumn{2}{|c|}{$\log I_{m}^{t_{L}}=0.10$} & \multicolumn{2}{|c|}{$\begin{array}{ll}t_{L} & =0.50 \\
\log I_{m} & \log \text { P.E.1 }\end{array}$} & \multicolumn{2}{|c|}{$\begin{aligned} t L & =0.90 \\
\log I_{m} & \log \text { P.E.1 }\end{aligned}$} \\
\hline 2 & & & $\overline{6} .6095$ & $\overline{7} .0405$ & $\begin{array}{l}\bar{\delta} .0686 \\
\bar{E} .0723\end{array}$ & $\begin{array}{l}\overrightarrow{7} .5487 \\
\overline{7} .5454\end{array}$ \\
\hline 4 & & & $\overline{6} .6736$ & $\overline{\overline{7}} .1897$ & $\overline{\overline{5}} .4216$ & $\overline{7} .8757$ \\
\hline 5 & & & $\overline{6} .9373$ & $\overline{7} .7479$ & $\begin{array}{l}\overline{\mathbf{b}} .5516 \\
\overline{\mathbf{b}} .5466\end{array}$ & $\begin{array}{l}\overline{6} .1567 \\
\overrightarrow{6} .0957\end{array}$ \\
\hline 7 & & & $\overline{6} .9346$ & $\overline{7} .3341$ & $\overline{\mathbf{5}} .8187$ & $\overline{6} .4270$ \\
\hline 8 & $\overline{6} .3649$ & $\overline{8} .8924$ & $\overrightarrow{\bar{b}} .2384$ & $\overline{6} .5384$ & $\begin{array}{l}\bar{\sigma} .9594 \\
\overline{5} .9996\end{array}$ & $\begin{array}{l}\overline{6} .4233 \\
\overline{6} .3734\end{array}$ \\
\hline 9 & & & & & $\overline{4} .2148$ & $\overline{6} .6676$ \\
\hline 10 & $\overline{\mathbf{6}} .6949$ & $\overline{7} .1411$ & $\overline{\tilde{\sigma}} .6763$ & $\overline{6} .1377$ & $\begin{array}{l}\overline{4} .4717 \\
\overline{4} .4768\end{array}$ & $\begin{array}{l}\overrightarrow{5} .1499 \\
\overline{6} .8742\end{array}$ \\
\hline 12 & $\overline{5} .0962$ & $\overline{7} .5690$ & $\overline{\bar{b}} .9848$ & $\overline{6} .4544$ & $\begin{array}{l}\overline{4} .9366 \\
\overline{4} .9349\end{array}$ & $\begin{array}{l}\overline{5} .5446 \\
\overline{5} .4200\end{array}$ \\
\hline 15 & $\overline{6} .7021$ & $\overline{6} .1747$ & $\overline{4} .6838$ & $\overline{5} .0917$ & $\begin{array}{l}\overline{3} .4493 \\
\overline{3} .4767\end{array}$ & $\begin{array}{l}4.1306 \\
4.0262\end{array}$ \\
\hline 18 & $\overline{4} .4195$ & $\overrightarrow{6} .9416$ & $\overline{3} .2765$ & $\overline{5} .7621$ & $\begin{array}{l}\overline{2} .1467 \\
\overline{2} .1235\end{array}$ & $\begin{array}{l}\overline{4} .6338 \\
\overline{4} .5924\end{array}$ \\
\hline 20 & $\overline{4} .7667$ & $\overline{5} .2744$ & $\overline{3} .6166$ & $\overline{5} .9389$ & $\begin{array}{l}\overline{\overline{2}} .4818 \\
\overline{\mathbf{2}} .4929 \\
\overline{2} .4729\end{array}$ & $\begin{array}{l}\overline{4} .9490 \\
\overline{4} .9477 \\
\overline{4} .9179\end{array}$ \\
\hline 22 & $\overline{4} .9610$ & $\overline{5} .3297$ & $\overline{3} .8228$ & $\overline{4} .2854$ & $\begin{array}{l}\overline{2} .7069 \\
\overline{2} .7207\end{array}$ & $\begin{array}{l}\overline{3} .2570 \\
\overline{3} .2527\end{array}$ \\
\hline 25 & $\overline{3} .2548$ & $\overline{5} .8353$ & $\overline{2} .1268$ & $\overline{4} .5204$ & $\begin{array}{l}\overline{2} .9932 \\
\mathbf{1} .0022\end{array}$ & $\begin{array}{l}\overline{3} .4692 \\
\overline{3} .4720\end{array}$ \\
\hline 30 & $\begin{array}{l}\overline{3} .7843 \\
\overline{\mathbf{3}} .7913\end{array}$ & $\begin{array}{l}\overline{4} .2037 \\
\overline{4} .2042\end{array}$ & $\overline{2} .6770$ & $\overline{3} .1989$ & i. 3801 & $\begin{array}{l}\overline{3} .8726 \\
\overline{3} .8497\end{array}$ \\
\hline 33 & & & $\overline{2} .9478$ & $\overline{3} .3516$ & $\overline{1} .7179$ & $\overline{2} .2809$ \\
\hline 35 & $\overline{\mathbf{2}} .2567$ & $\overline{4} .7288$ & 1.0934 & $\overline{3} .6108$ & $\begin{array}{l}\overline{1} .8988 \\
\overline{1} .9057\end{array}$ & $\begin{array}{l}\overline{2} .2872 \\
\overline{2} .3942\end{array}$ \\
\hline 38 & & & $\overline{1} .4495$ & $\overline{2} .0345$ & 0.2808 & 2.8894 \\
\hline 40 & $\overline{\mathbf{2}} .8010$ & $\overline{3} .4135$ & $\overline{1} .6660$ & $\overline{2} .1924$ & $\begin{array}{l}0.5603 \\
0.5470\end{array}$ & $\begin{array}{l}1.0728 \\
2.9529\end{array}$ \\
\hline 43 & & & 0.0697 & $\overline{2} .5342$ & 0.9619 & $\overline{\mathbf{1}} .5318$ \\
\hline 45 & $\overline{\mathbf{1}} .5326$ & $\overline{3} .9451$ & 0.4681 & $\overline{2} .8685$ & $\begin{array}{l}1.3294 \\
1.3440\end{array}$ & $\begin{array}{l}1.9665 \\
1.8679\end{array}$ \\
\hline 47 & & & 0.6931 & $\overline{1} .1354$ & 1.8013 & 0.2591 \\
\hline 48 & 0.2868 & $\overline{2} .6592$ & 1.2014 & $\overline{1} .7156$ & $\begin{array}{l}2.1970 \\
2.2201 \\
3.0287 \\
2.9605\end{array}$ & $\begin{array}{l}0.9468 \\
0.6583 \\
1.4591 \\
1.4342\end{array}$ \\
\hline $\begin{array}{l}50 \\
51 \\
52\end{array}$ & 1.5486 & $\overline{1} .8261$ & $\begin{array}{l}2.3454 \\
2.3420 \\
3.4087\end{array}$ & $\begin{array}{l}0.8464 \\
0.9756 \\
1.0384\end{array}$ & 2.9843 & 1.4817 \\
\hline
\end{tabular}


clues as to the physicochemical character of the primary excitation process. Mere subdivision of the illuminated area could not possibly change "reaction

TABLE II

Data for flicker contours obtained with a square image area totaling 9 square degrees, subdivided into four equal squares by vertical and horizontal cross-bars each $0.3^{\circ}$ wide; the center of the cross being $6^{\circ}$ on the temporal side of the fovea. These data are to be compared with those in Table I for the simple $3^{\circ} \times 3^{\circ}$ square all in one piece. Observer W. J. C., left eye; $n=10$.

\begin{tabular}{|c|c|c|c|c|c|c|}
\hline \multirow{2}{*}{$\frac{\begin{array}{c}F \\
\text { per sec. }\end{array}}{2}$} & \multicolumn{2}{|c|}{$\log I_{m}^{t L}=0.10$} & \multicolumn{2}{|c|}{$\log I_{m} \quad \stackrel{0.50}{\log \text { P.E.1 }}$} & \multicolumn{2}{|c|}{$\log I_{m}{ }^{0.90} \log$ P.E. } \\
\hline & $\overline{\mathbf{5}} .5896$ & $\overline{6} .0437$ & 4.0368 & $\overline{6} .5216$ & $\overline{4} .5362$ & $\overline{5} .0705$ \\
\hline 6 & $\overline{5} .8706$ & $\overline{6} .1621$ & 4.3535 & $\overline{6} .7251$ & 4.8963 & $\overline{5} .3624$ \\
\hline 10 & $\overline{4} .1544$ & $\overline{6} .5098$ & $\overline{4} .6482$ & $\overline{6} .9960$ & $\overline{3} .1878$ & $\overline{5} .6541$ \\
\hline 14 & 4.6731 & $\overline{5} .1187$ & $\overline{3} .0716$ & $\overline{5} .5204$ & $\overline{3} .6393$ & $\overline{5} .9576$ \\
\hline 16 & & & & & $\overline{3} .8075$ & $\overline{4} .2777$ \\
\hline 17 & $\overline{4} .9387$ & $\overline{5} .5105$ & & & & \\
\hline 18 & & & $\overline{\mathbf{3}} .6186$ & $\overline{4} .0338$ & $\overline{2} .1664$ & $\overline{4} .4564$ \\
\hline 19 & $\overline{\mathbf{3}} .4663$ & $\overline{5} .9983$ & $\overline{2} .0496$ & $\overline{4} .4241$ & $\overline{\mathbf{2}} .6256$ & $\overline{4} .9719$ \\
\hline \multirow[t]{2}{*}{20} & $\overline{\mathbf{3}} .7858$ & 4.1610 & 2.6606 & $\overline{4} .9877$ & $\overline{1} .3895$ & $\overline{3} .7798$ \\
\hline & $\overline{\mathbf{3}} .7932$ & $\overline{4} .1414$ & $\overline{\mathbf{2}} .6841$ & $\overline{4} .9999$ & $\overline{1} .4938$ & $\overline{2} .0034$ \\
\hline \multirow[t]{2}{*}{22} & $\overline{2} .4901$ & 4.9249 & 1. 0633 & $\overline{3} .5854$ & $\mathbf{1} .6647$ & $\overline{2} .1363$ \\
\hline & $\underline{\mathbf{2}} .4984$ & $\overline{4} .8552$ & 1.1018 & $\overline{3} .5762$ & $\underline{1} .6244$ & $\overline{2} .0608$ \\
\hline \multirow[t]{3}{*}{25} & $\overline{2} .9091$ & $\overline{3} .4289$ & $\underline{1.4816}$ & 3.9960 & $\overline{1} .8962$ & $\overline{2} .3080$ \\
\hline & & & 1.3226 & $\overline{3} .7931$ & & \\
\hline & & & $\overline{\mathbf{1}} .4112$ & $\overline{3} .7547$ & & \\
\hline \multirow[t]{3}{*}{30} & $\overline{1} .2251$ & $\overline{3} .6523$ & 1.8242 & $\overline{2} .1775$ & 0.1718 & $\overline{2} .6365$ \\
\hline & & & $\overline{1} .6628$ & $\overline{2} .0844$ & & \\
\hline & & & $\overline{\mathbf{1}} .6813$ & $\overline{2} .0173$ & & \\
\hline \multirow[t]{2}{*}{35} & $\overline{\mathbf{1}} .5678$ & $\overline{3} .9732$ & $\overline{1} .9635$ & $\overline{2} .1273$ & 0.4717 & $\tilde{2} .8669$ \\
\hline & & & 0.0146 & $\overline{2} .5026$ & & \\
\hline \multirow[t]{2}{*}{40} & $\overline{1} .8966$ & $\overline{2} .3267$ & 0.3827 & $\overline{2} .7324$ & 0.8227 & 1.2419 \\
\hline & & & 0.3683 & $\overline{2} .7843$ & & \\
\hline \multirow[t]{2}{*}{45} & 0.4712 & $\overline{2} .8844$ & 1.0082 & $\overline{1} .6089$ & 1.4786 & $\overline{1} .8508$ \\
\hline & & & 0.9528 & 1.3601 & & \\
\hline \multirow[t]{3}{*}{48} & 0.9999 & $\overline{1} .5367$ & 1.3675 & 1.6347 & 2.0461 & 0.4123 \\
\hline & & & 1.6210 & 1.8894 & & \\
\hline & & & 1.6217 & 0.0420 & & \\
\hline \multirow[t]{2}{*}{50} & 1.5181 & $\overline{1} .9547$ & 2.1603 & 0.7309 & 2.7521 & 1.3171 \\
\hline & & & 1.9760 & 0.4015 & & \\
\hline \multirow[t]{2}{*}{51} & 2.6747 & 1.1632 & 2.6747 & 1.1632 & & \\
\hline & & & 3.0448 & 1.5308 & & \\
\hline
\end{tabular}

orders." Table II includes at $t_{L}=0.90$ two sets of measurements made about a year apart. They show good agreement.

In correlation with the abrupt steepness of the photopic curves in Fig. 2, we note that $F_{\max }$. does not change so rapidly with $t_{L}$ (Fig. $3 b$ ), although the rate 


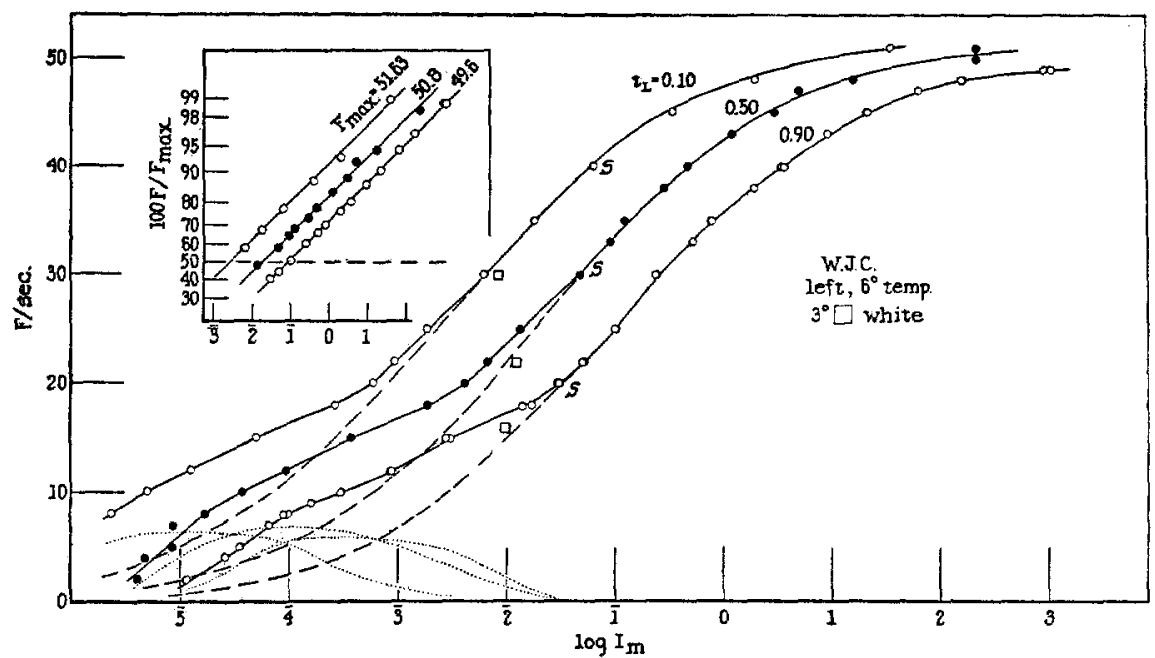

Fig. 1. $F-\log I$ contours for a $3^{\circ}$ square image, centered $6^{\circ}$ horizontally on the temporal side of the left fovea; white light; light-time fractions $t_{L}=0.10,0.50,0.90$. The inset figure shows the upper portions of these curves on a probability grid, computed to the values of $F_{\max }$. indicated; to show that the slope constants $\left(\sigma^{\prime} \log I\right)$ are identical. These probability integrals are shown extrapolated, below, and the separated "rod" contributions are given by the dotted lines. Data in Table I.

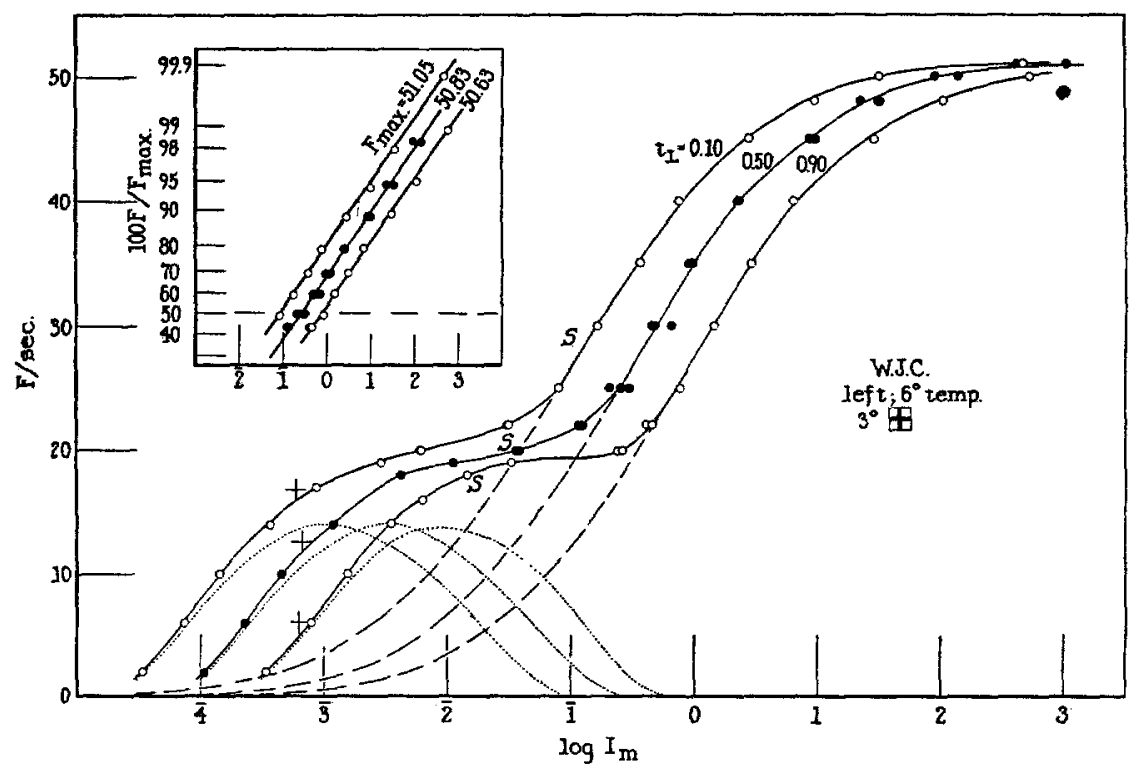

FIG. 2. Conditions as in Fig. 1, but here the $3^{\circ}$ square has been subdivided into four equal parts, separated by dark bars $0.3^{\circ}$ broad (see text). Data in Table II. In comparison to the curves in Fig. $1, \sigma^{\prime}{ }_{\log I}$ is less, but is again the same for the three values of $t_{L}$. The scotopic segments are much larger than in Fig. 1. The ascending and the descending branches of the separated-out "rod" components are probability integrals drawn with $F_{\max }=14.25,14.25,13.9$. 
of change of the abscissa of inflection $\tau^{\prime}$ (Fig. $3 a$ ) is about the same as for the undivided field of the same total illuminated area.

In general, above a certain small size, increase of image area $A$ in a given retinal region causes the "cone" curve to become steeper..$^{13}$ Comparisons with contours for image sizes larger and smaller than $3^{\circ} \times 3^{\circ}$, centered at $6^{\circ}$ from the fovea, show that the slope increases with $A$ in this way..$^{13}$ The interpretation that this is due to the involvement of a larger number of cone units is consistent with the facts that the slope constant $\sigma_{\log I}^{\prime}$ is $(a)$ independent of $t_{L}$ and of temperature, ${ }^{14}$ although a function of wave length; and $(b)$ it changes in the expected way when a given illuminated patch is placed at different locations on the retina where the numbers of cone units differ. The evidence is consid-

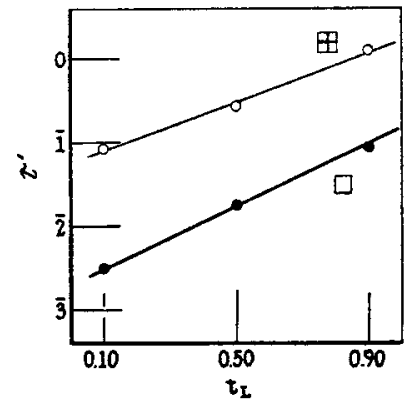

$a$

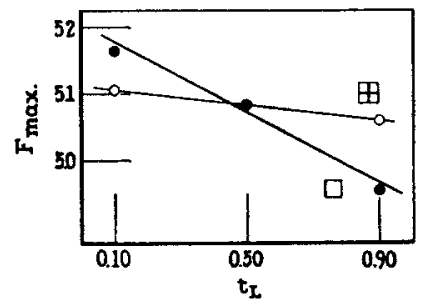

$b$

Frg. 3. The rectilinear relations of $\tau^{\prime}$ to $t_{L}$ and of $F_{\max }$. to $t_{L}$, are shown for the (photopic) $F-\log I$ contours of Figs. 1 and 2.

ered in some detail in a following paper. ${ }^{13}$ The argument for the necessity of dealing with all the available units at all levels of $F$ and $I$ has been set out previously. ${ }^{15}$

The question arises whether this can be the explanation for the difference between the "cone" slope constants in Figs. 1 and 2. We believe that it can. The flicker end-point is conceived to be brought about, neurally, by the production of a certain frequency of what we have termed "elements of effect;" the same end-point, namely recognition of flicker, can be achieved by a smaller average number of such elements from each of a larger number of neural units or by a greater mean contribution from each of a smaller number of units. Subjectively, at the end-point, all of the illuminated field, subdivided or not, is seen to flicker at once, but the effect is sharper at the boundaries. It is important to realize that the modification of the flicker contours with field (ii)

14 1937-38, J. Gen. Physiol., 21, 313; 1938-39, 22, 311; 1939-40, 23, 531; 1940-41, 24,$635 ; 1941-42,25,89,293,369 ; 1943-44,27,119$.

15 1943-44, J. Gen. Physiol., 27, 119. 
appears well below the brightness level at which there is perception of the fact that the field is actually subdivided (e.g., with $t_{L}=0.10$, the bars are not visible until a flash intensity of $\overline{4} .80 \mathrm{log} \mathrm{ml}$. units is reached). In comparison to the $3^{\circ}$ square field, the square broken into four parts has twice the perimeter of light/dark separation. The $F-\log I$ contours can be compared with those for a $6^{\circ}$ square, having very nearly the same perimeter as that of field (ii). The comparison is not altogether simple, since the shifts of $\tau^{\prime}$ with $t_{L}$ are not the

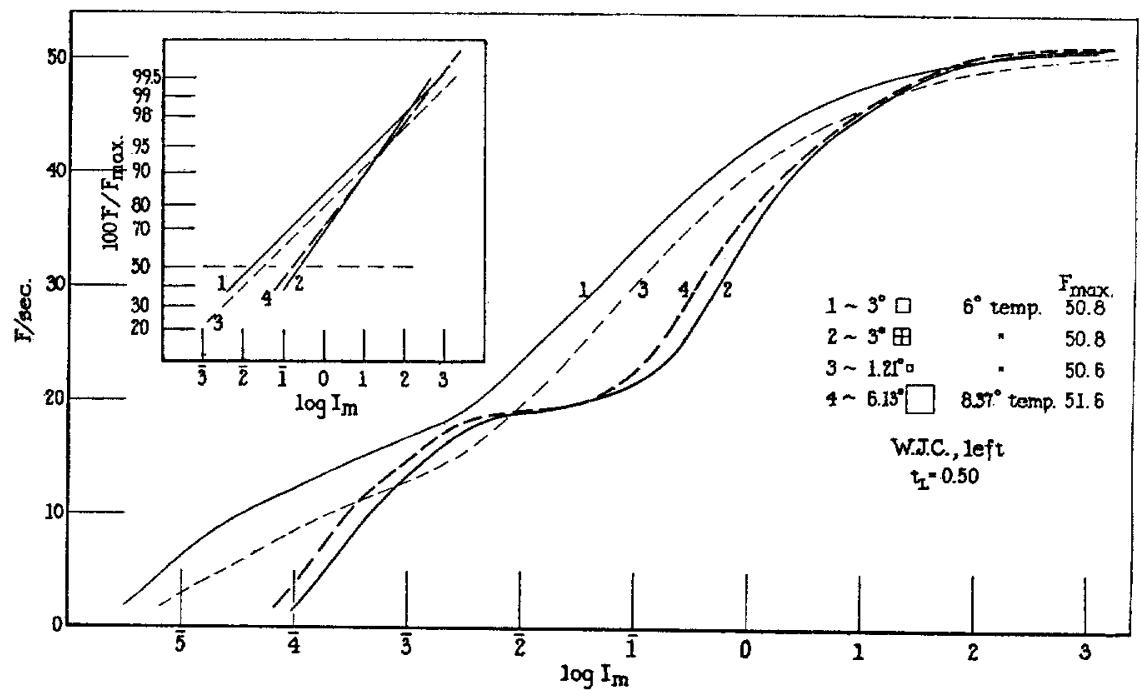

FIG. 4. Certain $F-\log I$ contours, $t_{L}=0.50$, for square images (white light), centered at $c a .6^{\circ}$ on the temporal side of the left fovea (W. J. C.), are traced for comparison. See text. The heavier continuous line for the $3^{\circ}$ square area subdivided into four equal parts approaches quite near to the heavier dashed line for the simple $6.13^{\circ}$ square (centered $8.4^{\circ}$ off the fovea; there is no real difference produced, for such a square, by the slightly more temporal centering, which, however, has for our purpose certain advantages).

The inset graphs show the upper parts of the main contours on a probability grid. See text.

same, but qualitatively the indications are suggestive (Fig. 4). Here we have traced the curves for several image areas at $t_{L}=0.50$. The contours for the $6^{\circ}$ and the subdivided $3^{\circ}$ squares are even closer together at $t_{L}=0.90 ; t_{L}=0.50$ was chosen because there the several values of $F_{\max }$. are closest together. Fig. 4 shows that one of the four parts of field (ii) gives a curve very little changed from that for the simple $3^{\circ}$ square (i), but that doubling the extent of the light/dark edge, keeping $A$ constant, has about the same effect as doubling the size of the square. The conception of a smaller mean contribution with (ii) 
from each excitable unit, that is a smaller frequency of elements of effect from each, is strengthened by the greatly reduced change of $F_{\max }$. with change of $t_{L}$.

This general conclusion as to the significance of the lines of light-dark separation is reinforced by the findings in the experiments of section $V$. It has already been stressed in another connection, namely in the data on the excitation of the eyes of bees; $;^{16}$ but there it is primarily a matter of the total frequency with which impulses are being generated, and the eye area illuminated at any instant is not the same when more stripes are introduced into a field. On the other hand, when checker-board fields are used, with $I$ the same, fields with the same black-white perimeter have the same excitatory value for the bee although $A$ is quite different.

The significance of contours in the flickered field, of course, introduces a somewhat novel factor into the interpretation of tests made in search of evidence for "summation" and the like, by placing one flickered patch near to another one. The most important feature of the situation may then be, not that the total illuminated area has been increased, but that there is an unilluminated zone between the patches. This is not to say that the image area is not a significant factor, however. The interesting points arising in relation to the rôle of the form of the image, and of the differential illumination of its several parts, we cannot now discuss. One approach to the question of the relative rôles played by total illuminated area on the one hand and on the other by image contour, is undertaken in section $\mathrm{V}$.

The dynamical interrelations of the groups of "rod" and "cone" effects in critical flicker have been discussed ${ }^{10}$ in terms of the partial inhibition of rod units by the activation of cone units, and the statistical summation in terms of probability integrals of the remainder with the cone effects. It follows from this conception that if the cone curve can be made steep enough, and moved to relatively higher intensities, the rising branch of the scotopic $F-$ $\log I$ curve could be completely freed from cone involvement. ${ }^{10}$ This we have found to occur normally in certain fishes. ${ }^{17}$ The effect has been produced in the human $F-\log I$ contour by imposition of several kinds of special conditions, ${ }^{18}$ but ordinarily the "rod" contribution must be extracted by subtraction of ordinates of the extrapolated "cone" curve.19 The present data on fields (i) and (ii) illustrate and justify these principles. It is apparent (Figs. 1 and 2) that with the subdivided field the scotopic branch of the con-

${ }^{16}$ Wolf, E., 1932-33, J. Gen. Physiol., 16, 773; Wolf, E., and Crozier, W. J., 1932-33' J. Gen. Physiol., 16, 787; Zerrahn, G. 1933, Z. vergleich. Physiol., 20, 117, 151; Wolf, E., and Zerrahn-Wolf, G., 1934-35, J. Gen. Physiol., 18, 853.

17 1937-38, J. Gen. Physiol., 21, 17; Proc. Nat. Acad. Sc., 1937, 23, 516; J. Gen. Physiol., 1938-39, 22, 463.

18 1941-42, J. Gen. Physiol., 25, 369; 1943-44, 27, 287.

${ }^{19}$ Cf. 1937-38, J. Gen. Physiol., 21, 203, 313; 1940-41, 24, 505; 1941-42, 25, 369. 
tour is greatly enlarged, although it falls at higher intensities. The analysis ${ }^{19}$ shown indicates that when the lower tail of the "cone" curve is caused to slip out from under the "rod" curve the latter increases in ordinate size, as expected. It cannot very well be supposed that the number of anatomical retinal rod units has been increased by the mere putting of crossbars on the field, or that their intensity thresholds have been profoundly increased thereby.

There are two general routes of escape from analytical difficulties created in such connections. One may suppose that the data are of neural origin, in the retina, central to the layer of primary receptors. Or it may be presumed that the quantitative properties of the data are of central [nervous origin, even with

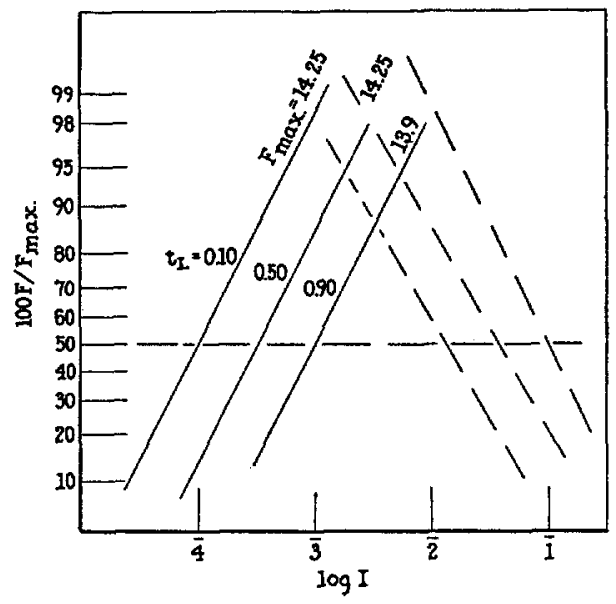

Fig. 5. The separated-out "rod" portions of the low intensity segments of the contours in Fig. 2, for the subdivided $3^{\circ}$ square, shown on a probability grid. (The rising branches are not so steep, i.e. $\sigma^{\prime}{ }_{\log I}$ is greater, in comparison to the corresponding features for the simple $3^{\circ}$ square in Fig. 1, signifying for the divided area an actual increase in functional number of "rod" units.)

monocular regard. There are good reasons for adopting the latter view, but they do not directly concern us now. It has been pointed out that "rod" curve flicker data, not "cone" complicated, exhibit certain significant properties which show them to be organically similar in basis of origin to the "cone" data. Thus the "rod" and "cone" curves in any one kind of animal are quantitatively shifted to the same, specific, extent as a function of change of temperature, ${ }^{20}$ and of change in $t_{L}{ }^{21}$ It is on these grounds quite impossible to assert that there is a different organic basis for the nature of "rod" effects on the one hand

${ }^{20}$ 1939, Proc. Nat. Acad.Sc., 25, 78, 171; 1938-39, J. Gen. Physiol., 22, 487; 193940, 23, 143.

${ }^{21} 1937-38$, J. Gen. Physiol., 21, 313; 1940-41, 24, 635. 
and "cone" effects on the other, as exhibited in the performance contours; or to suppose that different chemical mechanisms underly them. ${ }^{22}$ This evidence is consistent with the requirements of the experimentally determined fact that the resolution of the overlapping "rod" and "cone" contributions has now been demonstrated on a simple, uniform, statistical basis, under a variety of conditions, which would not be possible if the determination of the properties of the data did not occur in a common, simultaneous locus. The results of the analysis of the variation of critical intensities are in agreement with this position.

The dissected-out scotopic functional contributions to the $F-\log I$ contours of Figs. 1 and 2 are shown on a probability grid in Fig. 5. It is clear that

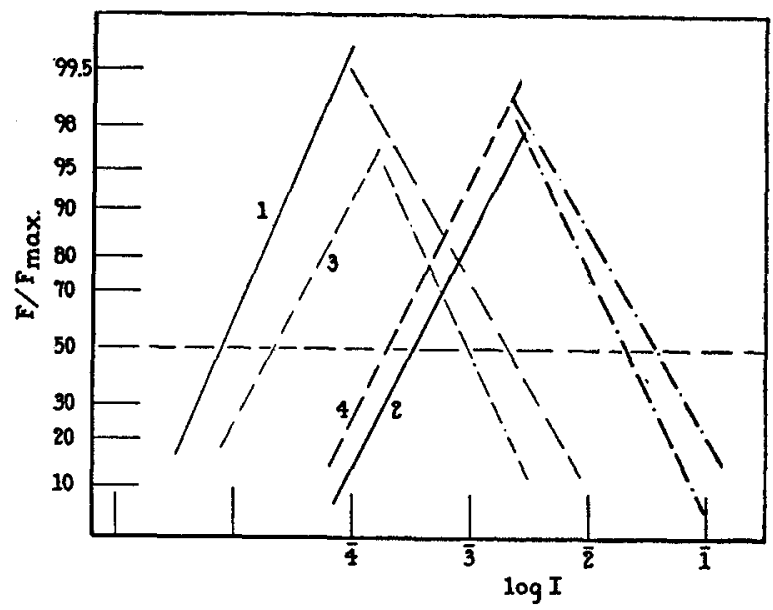

Fig. 6. Comparisons of the isolated "rod" components for the square images discussed in Fig. $4 ; t_{L}=0.50$.

the "rod" $\tau^{\prime}$ is again a rectilinear function of $t_{L}$; the proportionality constant is a little lower than for the "cone" branch, ${ }^{23}$ and slightly lower than for the plain $3^{\circ}$ square. From the comparisons in Fig. 4 it is apparent that the scotopic segment approaches that for the $6^{\circ}$ square, and the slope of the dissectedout "rod" curve does so likewise (Fig. 6).

IV

When flicker is produced by cutting a light beam at a focus, and repeated observations are made of the intensity $I_{c}$ critical for flicker, it is found in all our series of measurements that $\sigma_{1 x} \pm \sigma_{\sigma}$ is in direct, rectilinear proportion to $I_{m}$ over the whole range of $F$; but that in general the proportionality factor, and

22 1938, Proc. Nat. Acad. Sc., 24, 125; 1939, 25, 171.

${ }^{23}$ Cf. 1940-41, J. Gen. Physiol., 24, 635; 1941-42, 25, 89, 293, etc. 
$\sigma_{\sigma}$, are not independent of $A, \lambda, t_{L}$, the observer, or the eye used. All this means that the mechanism producing the data is one in which a high degree of organically determined internal correlation prevails. Part of the correlation found is due to the use of the same instrument and a reasonably fixed procedure, of course. If necessary, this factor could be experimentally identified and arithmetically extracted. But under our conditions systematic changes of the indices of variation as brought about by altering $t_{L}, \lambda$, or $A$, for example, ${ }^{24}$ or the form of the image, or by using monocular $v$. binocular regard, ${ }^{25}$ must be held to reflect properties of the observer in the system giving rise to the data.

The suggestion has been made ${ }^{26}$ that in "absolute" threshold measurements, and so possibly in data on discriminations at higher levels of intensity, the real source of the variability encountered is in the stimulating light delivered rather than in the observer. This idea first arose in connection with data on threshold stimulation by single brief flashes, ${ }^{27}$ where the possibility, if not indeed the significant actuality, of fluctuation in the number of quanta per single flash must be taken into account. The reality of any such consideration being required when trains of flashes are used (not shorter for the single flash than $c a .0 .009$ second), with fairly large image areas, at high intensities, can be ruled out. Moreover the experimental fact is that (as we have repeatedly shown) $\sigma_{1} / I_{m}$ is, for a given set of conditions, independent of intensity. But even for small, brief threshold flashes the argument proposed ${ }^{26}$ is unacceptable. For this there are two reasons: the description of the dependence of responsiveness upon $\Delta I_{0}$ by a Poisson summation ${ }^{26}$ is not unequivocal; and if it could be shown to be unique, it could not be referred to discrete fluctuations in the "external" stimulus at the retina.

The point of the second reason is, that if integral (Poisson) variations of external light quantity occur, the mean total number of quanta being small, then in each of the media of the retina, and at each interface between media, random (Poisson) quantal losses will be suffered by the bundle of quanta in each flash, through absorption, reflection, and scattering. Now it is a fact inherent in the mathematical properties of such situations that successive superimposed Poisson effects cannot add up to produce a final Poisson distribution with respect to successive flashes. Gaussian distributions do add up in this way, and are the only ones which can. ${ }^{28}$ Thus whether the frequency of positive threshold response is of Poisson form in terms of $\Delta I_{0}$, because the final number of available quanta is small, is one question; but if so it cannot be traced back to an original Poisson distribution in the initial flashes, at the retina, and consequently it cannot be said on any such basis that the essential variation in the data is due to fluctuations in the light rather than in the reacting organism.

${ }^{24} 1940-41$, J. Gen. Physiol., 24, 635; 1941-42, 25, 89, 293.

${ }^{25}$ 1940-41, J. Gen. Physiol., 24, 505.

${ }^{26}$ Hecht, S., Shlaer, S., and Pirenne, M. H., 1941-42, J. Gen. Physiol., 25, 819.

${ }^{27}$ Barnes, R. B., and Czernay, M., 1932, Z. Physik, 79, 436.

${ }^{28}$ Cremer, H., 1937, Random variables and probability distributions, Cambridge Tracts, Mathematics and Mathematical Physics, No. 35, London, Cambridge University Press. 
The remaining point has to do with the form of the distribution of the frequency of "successes" as a function of intensity near the threshold. The data specifically referred to in this discussion ${ }^{26}$ are actually better described by a probability integral in $\Delta I_{0}$ (not $\log I$ ), which accords with our own experience. It is specifically in agreement with the requirement of our view concerning the mode of origin of logarithmic frequency distributions in visual excitation, since for sufficiently short single flashes there is small opportunity for fluctuating performance in each excitable unit. ${ }^{6}$ Under these circumstances it is to be expected that nothing like the "reciprocity rule" for an inverse relation between exposure time and intensity critical for threshold should obtain, and extensive experimentation has shown us that it does not. Problems of the liminal photic energy for visual excitation have to be considered in terms of the properties of the assemblage of neural units involved.

It also accords with the meaning of results appearing when image area is varied, since then the mean liminal exciting quantity of light $\left(\Delta I_{0} \times A \times t\right)$ decreases with decrease of $\boldsymbol{A}$ down to a size so small that no exact image area can be estimated. Consequently, considerations of properties of threshold intensities in terms of energy and its fluctuations omit a factor vital to the whole situation. We therefore reject the suggestion that the fluctuations in critical intensities are not to be attributed to properties of the reacting organism.

The internal correlation manifest in the properties of $\sigma_{1_{I}}$ can be estimated directly, with reasonably homogeneous data. Since the mean value of the ratio $\sigma_{1} / I$ is independent of intensity, we can deal with the coefficients of variation collectively and write $\sigma_{1}$ for $\sigma_{1} / I$. For repeated tests with the same population sample it is known ${ }^{29}$ that

$$
\sigma_{\sigma}=\frac{\bar{\sigma}}{\sqrt{2 N}} \sqrt{1-r}
$$

where $\bar{\sigma}$ is the mean of all the S.D.'s, $\sigma_{\sigma}$ is the standard error of $\bar{\sigma}$, and $r$ is the coefficient of internal correlation. (This is, of course, usually employed for estimating $\sigma_{\sigma}$ when $r$ is known directly. Nothing in the derivation forbids reversing the argument to calculate $r$ when $\sigma^{2}$ is obtained from the data.) For coefficients of variation $V$ less than 10 per cent, as in the present case, $\sigma_{V}$ reduces to

$$
\sigma_{V}=\frac{\bar{V}}{\sqrt{2 r}},
$$

so that we can proceed with (1). Putting (1) into the form in which we deal with $\sigma$ 's for the distributions of $\sigma_{1} / I_{m}$, we have

$$
\sigma_{1_{1}}=\frac{\overline{\sigma_{1}}}{\sqrt{2}} \sqrt{1-r} .
$$

${ }^{29} C f$. Peters, C. C., and van Voorhes, W. R., 1940, Statistical procedures and their mathematical bases, New York and London, McGraw-Hill. 
It is this $r$ which we can use to obtain an invariant index of "organization" or integration among the neural units concerned in the determination of the flicker end-point along a given contour. It is independent of the level of $I$ of $F$, and thus of the level of photic adaptation. Its numerical values in the present series of experiments are quite high, but show a systematic although complex dependence upon $A, t_{L}, \lambda$, retinal location, and kind of image. In other types of tests of sensory discrimination its magnitude changes in a striking way. When experiments are deliberately contrived to introduce nonhomogeneity into the set of data considered, the value of $r$ drops toward zero. Thus, for a mixed set of three separated series of measurements of $\Delta I$ as a function of $I_{1}$ (W. J. C., left eye, field $12^{\circ} \times 12^{\circ}$, at the fovea, white light) we have $r=0.101$; whereas, for a single series $r=0.883$. In repeated independent series of flicker determinations the degree of agreement with respect to $r$ is indicated by such findings as these: $r=0.96,0.97$ ( $3^{\circ}$ field at $6^{\circ}$ temporal, white light, W. J. C., left eye); $r=0.98,0.98$, and $0.97,0.96$ for tests with our present field (iv). Under conditions as nearly alike as possible, $r$ for flicker, with an undivided field, changes in a characteristic way with $\lambda$, from 0.67 (violet) to 0.97 (green).

From data previously printed we may illustrate the kind of result which $r$ indicates. For uniocular and binocular flicker tests, with two observers, we were able to show ${ }^{25}$ that for the right and left eyes respectively the mean values of $\sigma_{1} / I_{m}$ were lower for the right eye than for the left, and still lower for binocular excitation. The significance of these facts for the theory of "binocular summation" has been discussed. ${ }^{25}$ It is of interest to consider the values of $r$ computed from these measurements $\left(6.13^{\circ}\right.$ square, centered at the fovea, white light, $\left.t_{L}=0.50\right)$ :

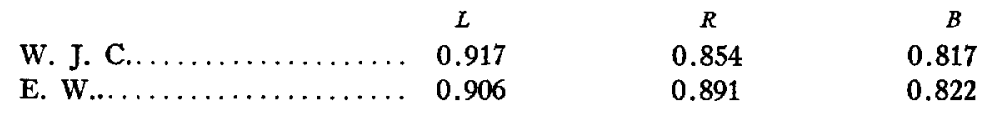

Thus, although the left eye ("dominant" in each case) gives a slightly higher value for $\sigma_{1} / I_{m}$ than the right, and both eyes together give a value lower in the ratio of $1: \sqrt{2}$ in the average, ${ }^{25}$ the "internal coherence" of the measurements, in terms of their exhibition of scatter, is slightly higher for the left and quite definitely lower for both eyes used simultaneously. (For auditory $L, R, B$ measurements $r$ behaves in a quite different way.) This result is consistent with the finding, to be set forth subsequently, ${ }^{13}$ that increase of flickered image area beyond a certain small size $\left(c a .1 .21^{\circ} \times 1.21^{\circ}\right)$ characteristically causes $r$ to drop. This may be complicated by the small but definite fall of $r$ which typically goes with increase of $t_{L}$. In general, when image area, or light-time fraction, or wave length, is increased $r$ rises to a maximum and then declines. A given series of measurements may fall on one or another branch of this kind of curve. In view of the correlated changes in $F_{\max }$, and in $\sigma_{\log I}^{\prime}$ this is 
taken to mean ${ }^{15}$ that $r$ is a function of the number of neural units involved and also of the density of elements of sensory effect they produce.

In a considerable number of series of measurements with various simple fields, on different parts of the retina, we find (as might be expected from the indications described in earlier papers ${ }^{30}$ ) that $r$ is a declining function of $\sigma_{\sigma}$. It has been shown that, when other things are equal, the scatter of $\sigma_{1}$ increases directly with the value of $F_{\max }$, and is thus a function of total number of elements of effect concerned. A test of this, which implies that in general $r$ should rise and then decline as $F_{\max }$. increases over a sufficiently wide range, is particularly interesting in the case of subdivided fields. In our "pecten effect" experiments already described ${ }^{3}$ there occurs a decrease of $\sigma_{\log I}^{\prime}$ as well as of $F_{\max }$. as $t_{L}$ is increased, signifying (in terms of our analysis) that both number of units acting and mean frequency of contributed elements of effect from each unit are altered when $t_{L}$ is varied. The curves for W. J. C. are pitched at lower intensities ( $\tau^{\prime}$ is smaller), are of lower $F_{\max }$, and $F_{\max }$. and $\tau^{\prime}$ change more extensively when $t_{L}$ is altered. This might lead to the conception that $r$ should increase with $t_{L}$ (and consequently with decline of $F_{\text {max. }}$.) for the W. J. C. data, which is found: $r$ rises steadily with fall of $F_{\max }$. for these curves, from $r=$ 0.68 to 0.86 . $F_{\text {max. }}$ for E. W. at $t_{L}=0.90$ is the same as for W. J. C. at $t_{L}=$ 0.10 , and the $r$ constants there agree $(0.86)$; but for E. W. they fall to 0.78 at $t_{L}=0.10\left(F_{\max .}=60.8\right)$.

Applying these considerations to the measurements with our $3^{\circ}$ subdivided square, where again $\sigma_{I}$ and $I_{m}$ are in rectilinear proportion, we find that the values of $\sigma_{\sigma}$ are consistently lower when the square is subdivided:

$\begin{array}{lllll} & t_{L} & =0.10 & 0.50 & 0.90 \\ 3^{\circ} \square & \log _{\sigma_{\sigma}} & =\overline{3} .755 & \overline{3} .867 & \overline{3} .883 \\ 3^{\circ} \text { 田 } & { }^{\circ} & =\overline{3} .697 & \overline{3} .738 & \overline{3} .639\end{array}$

while the values of $\bar{\sigma}$ may be a little lower, but not very significantly:

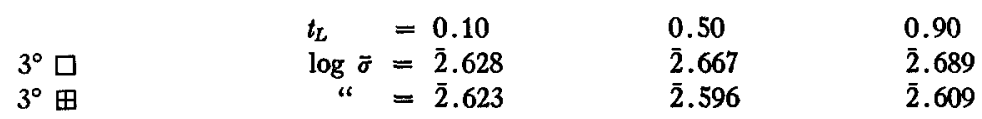

Thus, although the mean value of the precision with which the end-point is obtained is only slightly greater when the field is subdivided (by factors of $1.012,1.178$, and 1.202 as $t_{L}$ is made $0.10,0.50$, and 0.90 respectively), the scatter of $\sigma_{1} / I_{m}$ is decidedly lessened, by factors (in the same order) of 1.143 , $1.346,1.754$. We do not regard the almost rectilinear increase of these factors with $t_{L}$ to be necessarily accidental, but rather as pointing the way to a distinctly promising mode of inquiry.

The level of $F_{\max }$. (cf. Fig. 3) changes very little with $t_{L}$ for the subdivided

30 1940-41, J. Gen. Physiol., 24, 635; 1941-42, 25, 89, 293. 
square, and the values of $r$ do not change $(0.97,0.96,0.98)$; they seem a little higher than with the simple square of field (i), 0.96, 0.95, 0.96, but we cannot hold that such differences are significant. A wider range of tests is necessary before we can conclude that (in the range of $F_{\max }$. where, as in the experiments with fields (i) and (ii), $r$ is ordinarily at a maximum) an increase of the ("cone") slope constant without much change of $F_{\max }$. can affect the value of $r$. We can say, however, that for the same $\sigma_{\log I}^{\prime}$ the contours with field (iii), in section $\mathrm{V}$, and field (ii) give a higher value of $r$ for the field (ii) with lower $F_{\max }$.

It is clear that these variational constants provide one means of estimating in a simple way the influence of additional factors affecting the internal coherence of data in an otherwise homogeneous set. For example, if conditions are found which permit the comparison of flicker curves brought to the same $F_{\max }$. by choice of $t_{L}$, and to the same slope by choice of image area ${ }^{15}, r$ can be computed under the influence of lowered $\mathrm{O}_{2}$ pressure, medication, subdivision of the image, and the like. It should provide an index of the relative coherence of the state of neural integration governing the determination of the response. The properties of $r$ confirm in an independent way the multivariate character of the situation controlling visual end-points, and illustrate once more why it is futile to found interpretive conceptions upon data derived from any single set of "standard" circumstances.

\section{$\mathrm{v}$}

Systematic investigation of the origin of effects of the type discussed in section III has been inviting. Certain steps have been taken in this direction. ${ }^{13}$ Thus we have ascertained that the simple subdivision of a small field into two parts does not necessarily change the flicker contour if this is done in such a way as to produce only a small increase in the extent of the light/dark margin on the field. This could be pursued further. The promising but complex analytical possibilities presented by the flickering of a field in which parts are illuminated by one intensity while other parts are maintained at a different intensity (or $\lambda$ ) may be mentioned. A firm approach to questions posed by the Gestalt psychology of perception is, of course, thus possible.

We are now concerned, however, with the exploration of the nature of the "pecten effect" in flicker. ${ }^{3}$ The experiment discussed in section III has shown that mere subdivision of an illuminated field periodically illuminated by light sectored at a focus can cause the "cone" $F-\log I$ curve to be steeper, change its relation to the light-time fraction $t_{L}$ in the flash cycle, and alter its dependence of $F_{\max }$. on $t_{L}$. The simple splitting of the field (section III) does not, however, introduce into the dependence of the $F-\log I$ contour on $t_{L}$ the other major element of what we have recognized as the "pecten effect," namely the thorough upsetting of the normally simple, rectilinear dependence of $\tau^{\prime}$ on $t_{L}{ }^{3}$ This feature is, however, brought in, in a way which we have found sur- 
prising, by a further elaboration of the kind of experiment concerned in section III.

An illuminated field of $10^{\circ} \times 10^{\circ}$ boundary at the retina was crossed by vertical (or horizontal) stripes. The field was centered at the fovea. Two such fields, already referred to as (iii) and (iv), are mainly concerned here. On (iii) there were three dark and four light vertical bars, each $1.43^{\circ}$ wide. On (iv) there were six dark and seven light vertical bars, each $0.77^{\circ}$ wide. The

TABLE III

Data for flicker response contours with different light-time proportions, using a foveally centered squre test-field subtending $10^{\circ}$ on a side at the retina, but divided by three equally spaced vertical opaque bars $1.43^{\circ}$ wide, the four light bars thus produced being also $1.43^{\circ}$ broad. White light, W. J. C., left eye; $n=10$ at each point.

\begin{tabular}{|c|c|c|c|c|c|}
\hline $\begin{array}{c}F \\
\text { per } \\
\text { sec. }\end{array}$ & $\begin{array}{c}t_{L}=0.10 \\
\log I_{m} \log \text { P.E. } 1\end{array}$ & $\log I_{m}^{0.25} \log$ P.E. 1 & $\log I_{m} \stackrel{0.50}{\log \text { P.E.1 }}$ & $\log I_{m} \stackrel{0.75}{\log \text { P.E.1 }}$ & $\log I_{m}^{\text {p. } 90} \log$ P.E. \\
\hline $\begin{array}{l}2 \\
4\end{array}$ & & & $\begin{array}{l}\overline{6} .1900 \overline{8} .9123 \\
\overline{6} .4344 \overline{8} .8700\end{array}$ & & \\
\hline 6 & $\overline{\mathbf{7}} .4176 \overline{9} .8307$ & & $\overline{6} .7322 \overline{7} .0812$ & & $\overline{7} .9388 \overline{8} .4068$ \\
\hline 8 & $\overline{7} .7338 \overline{8} .1844$ & & $\overline{\mathbf{b}} .0249 \overline{\overline{7}} .3426$ & & $\overline{6} .2674 \overline{8} .6642$ \\
\hline 10 & $\overline{6} .0434 \overline{8} .4604$ & $\overline{6} .9098 \overline{7} .3605$ & $\overline{\overline{5}} .3606 \overline{\overline{7}} .7894$ & $\overline{6} .4251 \overline{8} .8221$ & $\overline{\mathbf{6}} .6029 \overline{7} .0178$ \\
\hline 12 & $\overline{6} .3406 \overline{8} .5274$ & & $\overline{\mathbf{b}} .6693 \overline{7} .9193$ & & $\overline{6} .8882 \overline{7} .0638$ \\
\hline 15 & $\overline{6} .5938 \overline{8} .9686$ & & $\overline{\mathbf{b}} .8991 \overline{6} .2488$ & & $\overline{\mathbf{b}} .1271 \overline{7} .3971$ \\
\hline 18 & $\overline{6} .9853 \overline{7} .0384$ & & $\overline{4} .3064 \overline{6} .6410$ & & $\overline{\mathbf{E}} . \overline{5350} \overline{6} .0799$ \\
\hline 20 & $\overline{\bar{b}} .2572 \overline{7} .5541$ & $\overline{4} .1467 \overline{6} .5627$ & 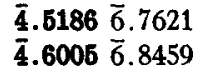 & $\overline{\mathbf{b}} .6690 \overline{6} .0889$ & 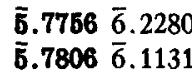 \\
\hline 25 & $\overline{\mathbf{b}} .6281 \overline{6} .0544$ & & $\overline{4} .9440 \overline{5} .2466$ & & $\overline{4} .1526 \overline{6} .5783$ \\
\hline 30 & $\overline{\tilde{5}} .9802 \overline{6} .3294$ & $\overline{4} .8710 \overline{5} .1851$ & $\overline{3} .2638 \overline{5} .6750$ & $\overline{4} .2830 \overline{6} .7527$ & $\overline{4} .6349 \overrightarrow{6} .8808$ \\
\hline 35 & $\overline{4} .3267 \overline{6} .7527$ & & $\overline{3} .6229 \overline{4} .0678$ & & $\overline{4} .8620 \overline{5} .3460$ \\
\hline 40 & $\overline{\mathbf{6}} .6985 \overline{6} .9240$ & $\overline{\mathbf{3}} .6544 \overline{5} .7266$ & $\overline{3} .9609 \overline{4} .6070$ & $\overline{3} .0338 \overline{5} .5013$ & $\overline{3} .2014 \overline{5} .6725$ \\
\hline 45 & $\overline{3} .0086 \overline{5} .2982$ & & $\overline{\mathbf{2}} .3066 \overline{4} .6791$ & & $\overline{\mathbf{3}} .6714 \underline{4} .0929$ \\
\hline 48 & $\overline{\mathbf{3}} .2667 \overline{5} .7830$ & & $\overline{\mathbf{2}} .6824 \overline{4} .9849$ & & $\overline{3} .8418 \overline{4} .1857$ \\
\hline 50 & $\overline{3} .6993 \underline{4} .0757$ & $\overline{\mathbf{2}} .4870 \overline{4} .8879$ & $\overline{2} .9034 \overline{3} .4385$ & $\overline{3} .9527 \overline{4} .4924$ & $\overline{2} .1861 \overline{4} .6021$ \\
\hline 52 & $\overline{3} .9862 \overline{4} .3947$ & & $\overline{1} .2931 \overline{3} .7900$ & & $\overline{\mathbf{2}} .6565 \overline{3} .0793$ \\
\hline 54 & $\overline{1} .3251 \overline{3} .8437$ & & $0.6188 \overline{1} .0810$ & & $\overline{1} .6068 \overline{3} .9226$ \\
\hline 55 & 1.58280 .0721 & & & & 3.09511 .6155 \\
\hline
\end{tabular}

idea was to have, within the same total extent of image field, about the sam ${ }^{\mathrm{e}}$ illuminated area but about twice the dark/light perimeter in one field as in the other. It will be understood that in an exact sense these several conditions cannot really be satisfied. But it will also be apparent that for the purposes of the present account they are satisfied to an approximation which is sufficient.

It appeared in the course of the observations that when $t_{L}$ was variously adjusted something peculiar was involved. This became the subject of very careful tests as to the possible existence of different kinds of flicker end-points 


\section{TABLE IV}

Data for flicker response contours with different light-time proportions using a square foveally centered test-field subtending $10^{\circ}$ on a side at the retina, but divided by six equally spaced vertical opaque bars $0.77^{\circ}$ wide, the seven light bars thus produced being also $0.77^{\circ}$ broad. White light, W. J. C., left eye; $n=10$ at each point.

\begin{tabular}{|c|c|c|c|c|c|}
\hline $\begin{array}{l}\text { per } \\
\text { sec. }\end{array}$ & $\begin{array}{c}t_{L}=0.10 \\
\log I_{m}=\log \text { P.E.I }\end{array}$ & $\log I_{m} \stackrel{0.25}{\log \text { P.E.1 }}$ & $\log I_{m}^{0.50} \log$ P.E. 1 & $\log I_{m} \stackrel{0.75}{\log \text { P.E. }}$ & $\log I_{m}{ }^{\text {p.90 }} \log$ P.E.s \\
\hline 2 & & $\overline{6} .3166 \overline{8} .7111$ & $\begin{array}{l}\overline{6} .6566 \overline{8} .8993 \\
\overline{6} .6427 \overline{8} .8284\end{array}$ & & $\overline{6} .1430 \overline{8} .3939$ \\
\hline $\begin{array}{l}4 \\
5\end{array}$ & & $\overline{6} .6527 \overline{8} .8319$ & $\begin{array}{l}\overline{6} .9909 \overline{7} .2377 \\
\overline{5} .1473 \overline{7} \cdot 4032\end{array}$ & $\overline{6} .0820 \overline{8} .4342$ & $\overline{\mathbf{6}} .3836 \quad \overline{8} .7004$ \\
\hline 6 & $\overline{6} .0265 \overline{8} .2884$ & $\overline{\mathbf{6}} .9117 \overline{7} .5147$ & $\overline{\mathbf{5}} .2891 \overline{7} .5574$ & $\overline{6} .3901 \overline{8} .7887$ & $\overline{\mathbf{6}} .6766 \overline{\overline{7}} .0489$ \\
\hline 8 & $\overline{6} .3390 \overline{8} .6692$ & & $\overline{\bar{b}} .6747 \overline{7} .7601$ & $\overline{6} .7232 \overline{7} .1889$ & $\overline{6} .9923 \overline{7} .1615$ \\
\hline 9 & & & $\overline{\mathbf{5}} .7813 \overline{6} .0305$ & & \\
\hline 10 & $\overline{6} .6980 \overline{7} .0014$ & $\overline{\mathbf{5}} .6909 \overline{6} .1366$ & 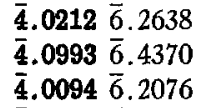 & $\overline{\bar{b}} .0715 \overline{7} .4736$ & $\overline{\bar{\sigma}} .3641 \overline{7} .6642$ \\
\hline 12 & $\overline{\mathbf{6}} .9699 \overline{7} .2736$ & $\overline{\mathbf{5}} .9602 \overline{6} .3093$ & $\begin{array}{l}\overline{4} .3688 \overline{6} .6823 \\
\overline{4} .2591 \quad \overline{6} .5297\end{array}$ & $\overline{\mathbf{b}} .3243 \overline{7} .6220$ & $\overline{\mathbf{b}} .6325 \overline{7} .9719$ \\
\hline 15 & $\overline{\mathbf{b}} .3183 \overline{7} .6981$ & $\overline{4} .2955 \overline{6} .7096$ & 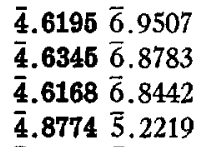 & $\overline{5} .6829 \overline{6} .0879$ & $\overline{\overline{5}} .9820 \overline{6} .4078$ \\
\hline 18 & $\overline{\mathbf{5}} .7124 \overline{6} .0083$ & $\overline{4} .7033 \overline{5} .2473$ & 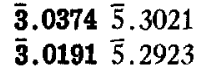 & $\overline{4} .0842 \overline{6} .5098$ & $\overline{4} .3820 \overline{6} .6847$ \\
\hline 20 & 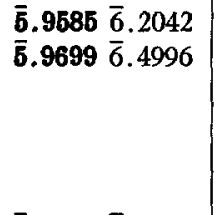 & $\begin{array}{l}\overline{4} .9405 \overline{5} .3635 \\
\overline{4} .9138 \overline{5} .2884\end{array}$ & $\begin{array}{ll}\overline{3} .2925 & \overline{5} .5387 \\
\overline{3} .2595 & \overline{5} .4550 \\
\overline{3} .2579 & \overline{5} .6329 \\
\overline{3} .2993 & \overline{5} .5498 \\
\overline{3} .2472 & \overline{5} .5122\end{array}$ & $\begin{array}{l}\overline{4} .3632 \overline{6} .5387 \\
\overline{4} .3841 \overline{6} .5584\end{array}$ & $\begin{array}{ll}\overline{4} .6221 & \overline{6} .7950 \\
\overline{4} .6294 & \overline{6} .9695\end{array}$ \\
\hline 25 & 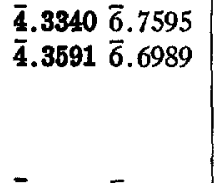 & $\begin{array}{l}\overline{3} .2655 \overline{5} .5813 \\
\overline{3} .2653 \\
\overline{5} .6839\end{array}$ & $\begin{array}{ll}\overline{3} .6965 & \overline{5} .9429 \\
\overline{3} .6145 & \overline{5} .9546 \\
\overline{3} .6142 & \overline{5} .8637 \\
\overline{3} .6188 & \overline{4} .0330\end{array}$ & $\begin{array}{l}\overline{4} .7423 \overline{5} .0857 \\
\overline{4} .7570 \\
\overline{5} .1854\end{array}$ & $\begin{array}{ll}\overline{3} .0441 & \overline{5} .3861 \\
\overline{3} .0693 & \overline{5} .4108\end{array}$ \\
\hline 30 & $\overline{4} .7560 \overline{5} .1824$ & $\overline{3} .6502 \overline{5} .9995$ & 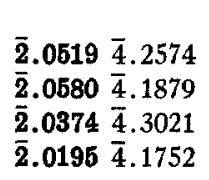 & $\overline{3} .1007 \overline{5} .5098$ & $\overline{3} .3936 \overline{5} .7681$ \\
\hline 35 & $\overrightarrow{3} .1222 \overrightarrow{5} .3001$ & $\overrightarrow{\mathbf{2}} .0077 \overrightarrow{4} .4159$ & $\begin{array}{ll}\overline{2} .3802 & \overline{4} .7288 \\
\overline{2} .3829 & \overline{4} .6311 \\
\overline{2} .4591 & \overline{4} .8096\end{array}$ & $\overline{3} .4673 \overline{5} .8637$ & $\overline{3} .7553 \overline{4} .0038$ \\
\hline 40 & 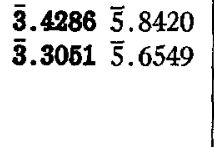 & 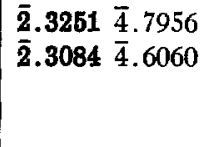 & $\begin{array}{ll}\overline{2} .7171 & \overline{3} .0094 \\
\overline{2} .7254 & \overline{3} .0513 \\
\overline{2} .7142 & \overline{3} .0506 \\
\overline{2} .7101 & \overline{3} .0038\end{array}$ & $\begin{array}{l}\overline{3} .7918 \overline{4} .1658 \\
\overline{3} .7824 \\
\overline{4} .2599\end{array}$ & $\begin{array}{ll}\overline{\mathbf{2}} .0338 & \overline{4} .4063 \\
\overline{\mathbf{2}} .0599 & \overline{4} .4468\end{array}$ \\
\hline
\end{tabular}


TABLE IV-Continued

\begin{tabular}{|c|c|c|c|c|c|}
\hline $\begin{array}{l}F \\
\text { per } \\
\text { sec. }\end{array}$ & $\begin{array}{c}t_{L}=0.10 \\
\log I_{m} \log \text { P.E. } 1\end{array}$ & $\log I_{m}^{0.25} \log$ P.E. 1 & $\log I_{m} \stackrel{0.50}{\log \text { P.E. } 1}$ & $\log I_{m} \stackrel{0.75}{\log \text { P.E.1 }}$ & $\log I_{m} \stackrel{0.90}{\log \text { P.E. } 1}$ \\
\hline 45 & $\overline{3} .7416 \overline{4} .1518$ & $\overline{\mathbf{2}} .6302 \overline{4} .9781$ & 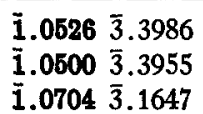 & $\overline{2} .1486 \overline{4} .6041$ & $\overline{\mathbf{2}} .405 \overline{4} .7208$ \\
\hline 48 & $\overline{3} .9517 \overline{4} .2028$ & $\overline{\mathbf{2}} .7811 \overline{3} .0468$ & $\begin{array}{ll}1.2499 & \overline{3} .5530 \\
\overline{1} .2646 & \overline{3} .5773\end{array}$ & $\overline{2} .3866 \overline{4} .7601$ & $\overline{\mathbf{2}} .7186 \overline{3} .0678$ \\
\hline 50 & $\overline{\mathbf{2}} .2997 \overline{4} .7345$ & $\overline{1} .0484 \overline{3} .4123$ & 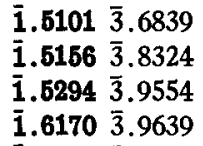 & $\overline{2} .6503 \overline{4} .9202$ & $\overline{2} .9983 \overline{3} .3512$ \\
\hline 52 & $\overline{\mathbf{2}} .6157 \overline{4} .8669$ & $\overline{\mathbf{1}} .4643 \overline{3} .8078$ & $\begin{array}{ll}\overline{1} .9312 & 2.3438 \\
\overline{1} .9024 & \overline{2} .1994\end{array}$ & $1.0792 \overline{3} .3301$ & $\overline{1} .4041 \overline{3} .7520$ \\
\hline 53 & & & $0.3008 \overline{2} .5454$ & $1.9054 \overline{2} .3228$ & 0.74531 .5432 \\
\hline 54 & $\overline{\mathbf{1}} .0550 \overline{3} .3581$ & $0.3740 \overline{2} .6791$ & $0.6678 \overline{1} .0812$ & 3.29041 .5874 & \\
\hline 55 & 0.47942 .9032 & $1.3128 \overline{1} .3301$ & 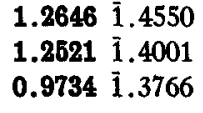 & & \\
\hline
\end{tabular}

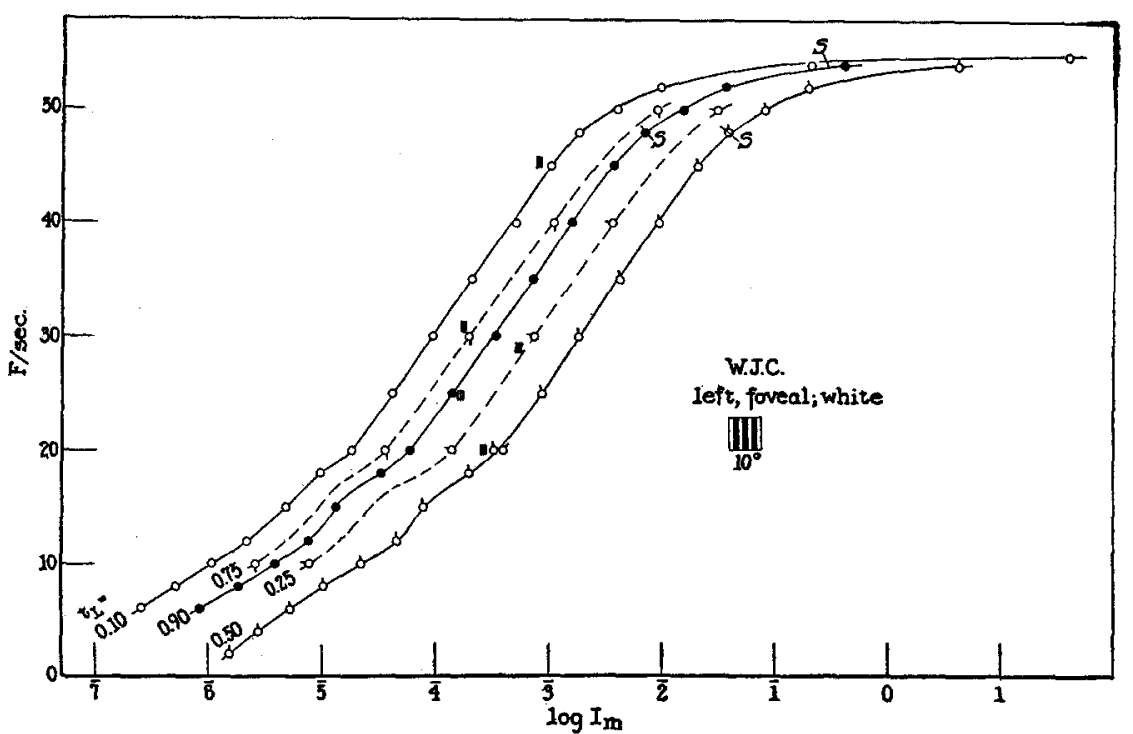

Fig. 7. Flicker contours for a square field $10^{\circ}$ on a side over-all, but subdivided into seven vertical bars $1.43^{\circ}$ wide alternately illuminated and opaque; centered at the fovea. Note that the curves for $t_{L}=\mathbf{0 . 7 5}$ and 0.90 are at lower intensities than that for $t_{L}=0.25$. The contours are quite steep, but clearly of the same general slope. The 3-bar symbol on each curve indicates the level at which the field was recognized as barred. See text. Data in Table III. 
which might be confused. We shall return to this presently. But it became clear that this kind of complication did not really enter. The peculiarity had to do predominantly with the location on the $\log I$ axis of the contours at $t_{L}=$ 0.75 and 0.90 . Precisely as in our experiments with moving vertical stripes on a barred field, the contours for $t_{L}=0.75$ and 0.90 were shifted bodily to much lower positions on the intensity scale, but in the present case with no change of slope constant and with very slight change of $F_{\max }$. This completes the objective evidence justifying the view that at least three independent parameters are required for the formulation of the (simplex) flicker curve, ${ }^{6}$ since each of our $\tau^{\prime}, \sigma_{\log I}^{\prime}$, and $F_{\max }$. parameters can thus be experimentally changed in uncorrelated ways. It also appears that three such parameters are sufficient.

The measurements for the contours with fields (iii) and (iv) are collected in Tables III and IV, and are shown in Figs. 7 and 8. The curves for the more finely subdivided field are pitched at a higher intensity level ( $\tau^{\prime}$ nearly $1 \log$ unit greater for field (iv) - cf. Fig. 10), but show qualitatively the same sort of dependence on $t_{L}$. $F_{\max }$. changes more with (iv) as $t_{L}$ is increased. As shown in Fig. 9 the slope constant $\sigma_{\log x}^{\prime}$ for (iv) is less than for (ii); i.e., the slope is greater. Here, as in section III, this automatically brings about an increase in the size of the "rod" contribution in (iv), in fact nearly doubles it, although it appears at a higher intensity ( $c f$. Figs. 1 and 2). Again we point out that such relationships cannot be rationalized in simple terms of an available population of illuminated retinal receptor units with individually fixed intensity thresholds. In (iv) the illuminated image area (52.9 square degrees) is actually less than that (57.2 square degrees) for (iii), although $\sigma_{\log I}^{\prime}$ is greater, so the "rod" effect cannot be accounted for on this basis.

The lengths of lines of separation between light and dark portions of the barred fields, however, are: (iii) $=91.44^{\circ}$, (iv) $=150.78^{\circ}$, in the ratio $1 / 1.65$. The "cone" slope constants $\left(1 / \sigma_{\log I}^{\prime}\right)$ are in the ratio $1 / 1.2$. In the experiment of section III, doubling the dark/light perimeter increases the slope in the ratio $1 / 1.43_{5}$.

The very considerable increase in ("cone") slope produced by increasing the subdivision of the field is not confined to white light, but is shown also with separated portions of the spectrum. Data with blue and red lights are given in Fig. 11 (Table V), for $t_{L}=0.50$. We have pointed out that with simple fields $F_{\max }$ and $\tau^{\prime}$ occupy an intermediate position for a white, as contrasted with the positions (on the same brightness or energy-at-cornea scale for $\tau^{\prime}$ ) taken by the opposite ends of the spectrum filtered from it. ${ }^{31}$ The properties of $\sigma_{\log I}^{\prime}$ for different spectral regions are not inconsistent with the view that white is not a simple "addition" of primaries, but represents a sort of integrative synthesis. ${ }^{31}$ In general, for simple fields, the $F-\log I$ contour at the same $t_{L}$ is with white

31 1941-42, J. Gen. Physiol., 25, 89, 293; 1943-44, 27, 119. 
intermediate $\left(\tau^{\prime}\right)$ between that for blue and red. The data of Fig. 11 show that by suitable subdivision of the test-field this order can be radically disturbed. On a scale of brilliance intensity the steady light values for the flash intensities adequate to evoke flicker with blue, red, white assume a different order when the field is suitably subdivided. This is also true if the intensities are put on an

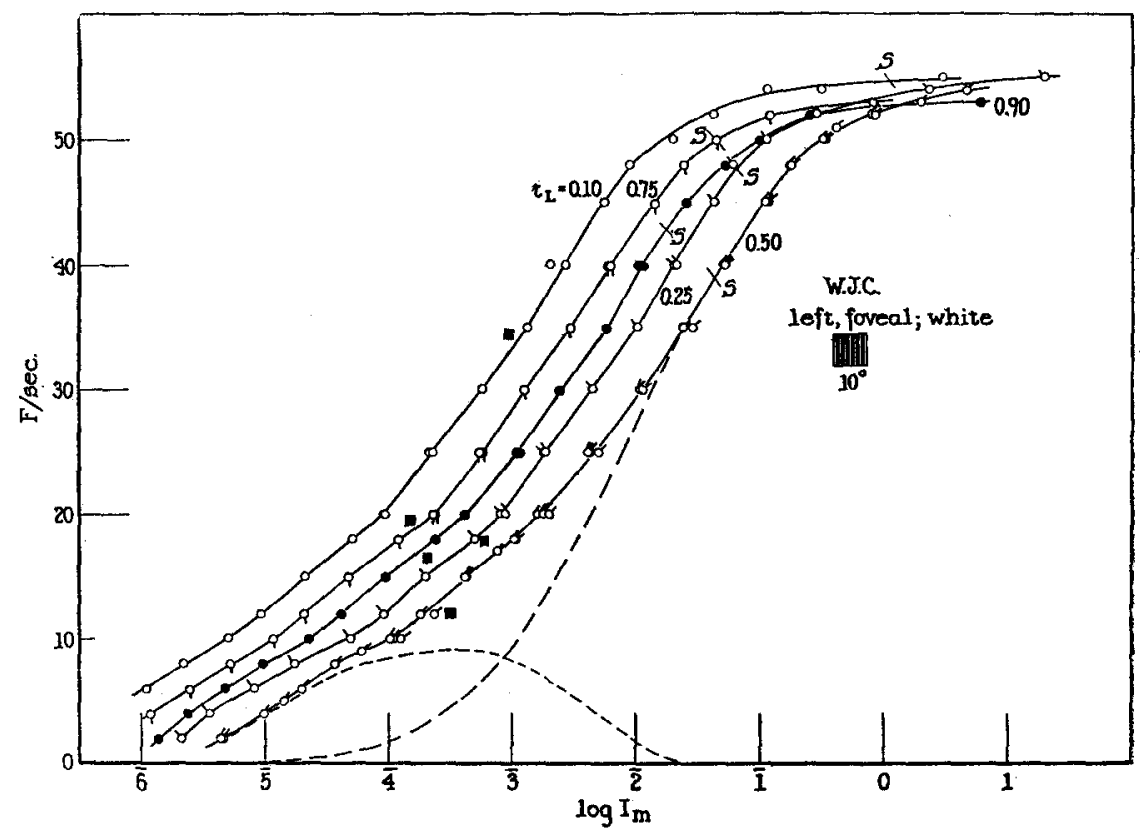

FIG. 8. As in Fig. 7 , a $10^{\circ}$ square field centered at the fovea, but divided into thirteen alternating light and dark vertical bars $0.77^{\circ}$ wide. The curves are steeper than those of Fig. 7 (cf. Fig. 9). Data in Table IV. The contours at $t_{L}=0.75$ and 0.90 are, as in Fig. 7, so shifted that they fall between those for $t_{L}=0.10$ and 0.25 . By comparison with the curves of Fig. 7 those here shown are located at higher intensity levels ( $c f$. Fig. 10). Above $\log I=c a . \overline{4} .6$, the illuminated fields are speckled. The intensities (barred symbol) at which the barred pattern is resolved, on the contour, are, however, about the same as in Fig. 7. At and above the flash intensities marked $S$ the illuminated bars are smooth.

energy basis. At this particular value of $t_{L}(=0.50), r$ for $B, W, R$ is respectively $0.99,0.97,0.95$; the differences in $\sigma_{\sigma}$ are more impressive: $\overline{3} .372, \overline{3} .699$, $\overline{3} .784$. These, like the foregoing, agree with the apparent order of $F_{\max .}$, while the differences detectable in $\sigma_{\log I}^{\prime}$ are insignificant. It is not without value for the conception of the multivariate control of the quantitative properties of the flicker contour that comparatively simple subdivision of the field should alter the order of relative effectiveness of different wave length zones. 
According to the view required by the data of the flicker contours we have supposed that (section III) a primary part may be played by the existence of lines of separation between light and dark areas in the flickered field. The striking modifications in the $F-\log I$ curves by simple subdivision of the image area are consistent with this notion. We have been required to assume that the existence of contrast edges, even when not visually resolved, serves to enhance the number of neural units concerned in the discrimination of

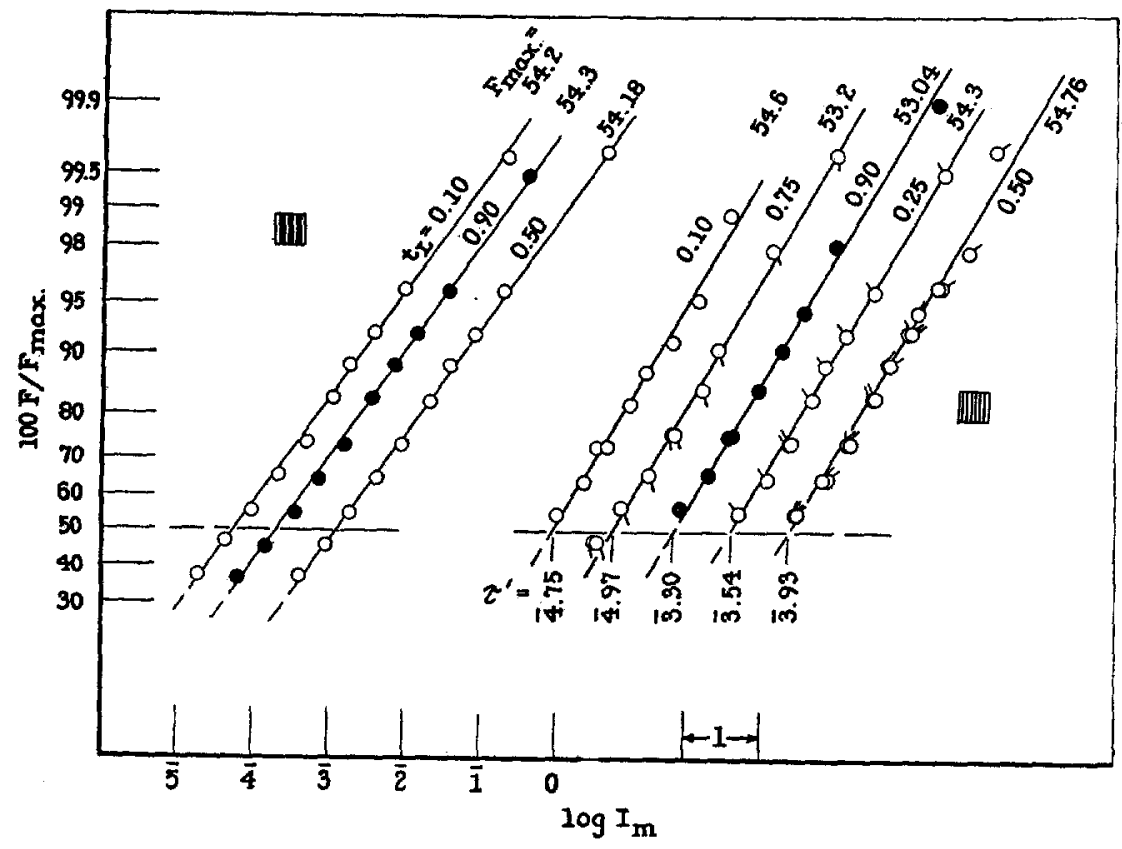

Frg. 9. The higher intensity segments of $F-\log I$ contours in Figs. 7 and 8, shown on a probability grid. Only the upper 50 per cent or so is uncomplicated by the "rod" contribution, but it is significant that for each set the slopes are uniform.

flicker, although total illuminate area is the same, and even though the whole field concerned subjectively flickers uniformly at the critical intensity.

In the data of our fields (iii) and (iv) it has to be emphasized that, as in the case of field (iii) (section III), the effect in question is definitely at work at flash intensities, let alone at brightnesses-at-fusion, well below those adequate for subjective recognition of the fact that the field is actually subdivided. At the moment, there are two chief aspects of interest in this situation. The first has to do with the argument for the essential unity of the organic mechanism determining the form of the flicker contour despite the manifestations of visual duplexity. We have already spoken of the importance of this conception as 


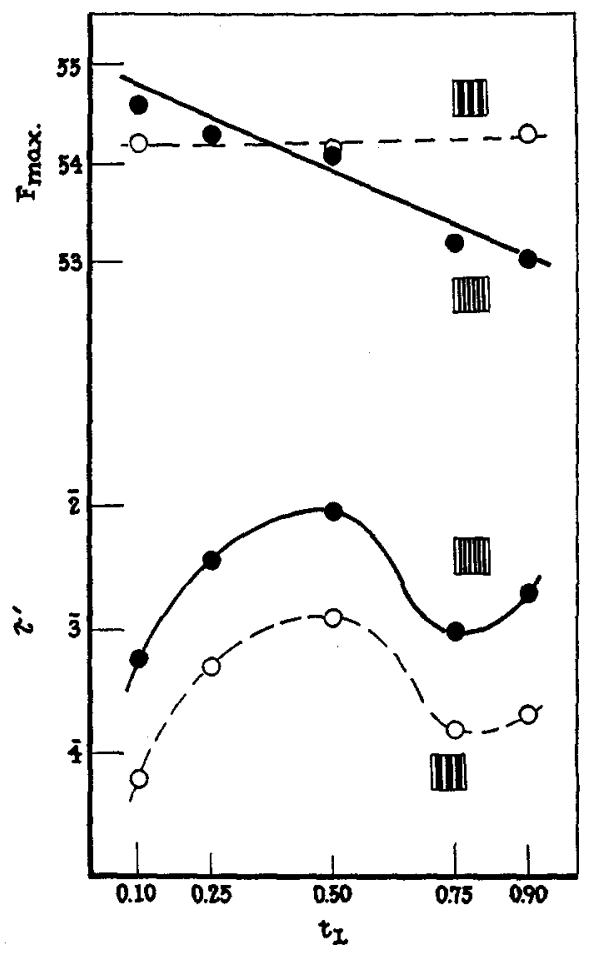

Fig. 10. The values of $F_{\max }$ and of the abscissa of inflection $\tau^{\prime}$ obtained from Fig. 9 are shown as functions of $t_{L}$, for the curves of Figs. 7 and 8. The structure of the flickered fields is indicated by the barred symbols.

TABLE V

Conditions as in Table IV, but using red light (two independent series) and blue; $t_{L}=$ 0.50. (Intensities in millilamberts, by matches with white.)

\begin{tabular}{|c|c|c|c|c|}
\hline \multirow{2}{*}{$\begin{array}{c}F \\
\text { per sec. }\end{array}$} & \multicolumn{2}{|c|}{$t_{L}=0.50$} & \multicolumn{2}{|c|}{$t_{L}=0.50$} \\
\hline & $\log I_{m}$ & log P.E.1 & $\log I_{m}$ & $\log$ P.E.1 \\
\hline 20 & $\begin{array}{l}\overline{4} .6416 \\
\overline{4} .6598\end{array}$ & $\begin{array}{l}\overline{5} .1117 \\
\overline{6} .9748\end{array}$ & $\overline{\bar{b}} .1172$ & $\overline{7} .4667$ \\
\hline 25 & $\begin{array}{l}\overline{3} .0020 \\
\overline{4} .9983\end{array}$ & $\begin{array}{l}\overline{5} .4241 \\
\overline{5} .2916\end{array}$ & $\overline{\mathbf{b}} .5097$ & $\overline{7} .9051$ \\
\hline 30 & $\begin{array}{l}\overline{3} .4240 \\
\overline{3} .4309\end{array}$ & $\begin{array}{l}\overline{5} .7272 \\
\overline{5} .8960\end{array}$ & $\overline{\mathbf{b}} .9501$ & $\overline{6} .2187$ \\
\hline 35 & $\begin{array}{l}\overline{\mathbf{3}} .8197 \\
\overline{\mathbf{3}} .8101\end{array}$ & $\begin{array}{l}\overline{4} .1343 \\
\overline{4} .3240\end{array}$ & $\overline{4} .2758$ & $\overline{6} .5901$ \\
\hline 40 & $\begin{array}{l}\overline{2} .1019 \\
\overline{2} .1866\end{array}$ & $\begin{array}{l}\overline{4} .3261 \\
\overline{4} .5074\end{array}$ & $\overline{4} .6397$ & $\overline{6} .8716$ \\
\hline 45 & $\begin{array}{l}\overline{2} .4295 \\
\overline{2} .4368\end{array}$ & $\begin{array}{l}\overline{4} .8989 \\
\overline{4} .9247\end{array}$ & $\overline{4} .8576$ & $\overline{5} .2005$ \\
\hline 48 & $\overline{2} .6639$ & $\overline{3} .0714$ & $\overline{3} .0820$ & $\overline{5} .4354$ \\
\hline 50 & $\overline{2} .9565$ & $\overline{3} .1484$ & $\overline{\mathbf{3}} . \mathbf{3 6 6 9}$ & $\overline{5} .6639$ \\
\hline 52 & $\overline{1} .4466$ & $\overline{3} .9721$ & $\overline{\mathbf{3}} .7820$ & $\overline{5} .1351$ \\
\hline 54 & 0.2972 & 2.7095 & & \\
\hline 55 & & & $\overline{1} .3418$ & $\overline{3} .7976$ \\
\hline
\end{tabular}


rationalizing the quantitative behavior of the scotopic section of the curve under various conditions both mild and drastic. The second aspect of theoretical importance concerns the relations of flash intensity, flash brightness, and especially of fused (Talbot) brightness in flickered light to "visual acuity." This is important in a number of ways which should have been examined a good while ago but seem to have been ignored. We are concerned here to indicate that the data provide a method for the experimental separation of effects due to subjective brightness from those due simply to intensity. Thus, on the curves of our "pecten" experiment ${ }^{3}$ the cross-barring of the field was perceptible at the same flash intensity down to a light-time fraction of 0.25 (E. W.) or 0.50

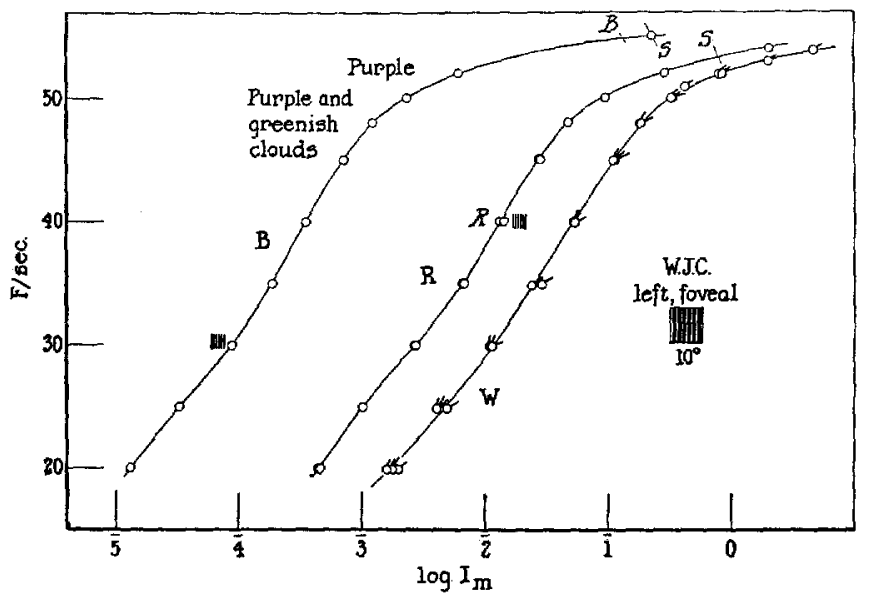

FIG. 11. Flicker contours, $t_{L}=0.50$, with the $10^{\circ}$ square subdivided into seven illuminated strips, centered at the fovea, for blue and red (Table V), compared with white on the same (steady light) brightness scale. The points at which the illumined fields become subjectively smooth, exhibit color, and are visually resolved as barred, are indicated.

(W. J. C.). In the experiment of section III the bars subdividing the field were just visible (on the contours) at a flash intensity of $\overline{4} .80 \mathrm{log}$ units (millilamberts), regardless of the light-time fraction, and thus independent of the fused brightness. (Incidentally, for $t_{L}>0.50$, this means that in the tests with the moving stripes ${ }^{3}$ the field is resolved at the same flash intensity regardless of whether there is any sign of involvement of the "cone" curve.) Consequently, we are dealing primarily with effects due to the physical intensity of a flash, rather than to its duration or to the subjective brightness level. For comparatively simple fields, the constancy of the flash intensity for visual resolution in flickered light ${ }^{32}$ extends to light-time fractions below 0.10 , although for more

${ }^{32}$ 1943-44, J. Gen. Physiol., 27, 119. 
complex fields this constancy may fail somewhat below $t_{L}=0.25$, while even so not obeying the course of the Talbot brightness. A more extensive examination of this matter has been made, and will be described elsewhere. Fields (iii) and (iv), with four and seven illuminated vertical bars respectively, were visually resolved (on the $F-\log I$ contours) at a $\log$ flash intensity which declined rectilinearly with increasing $t_{L}$ (Fig. 12).
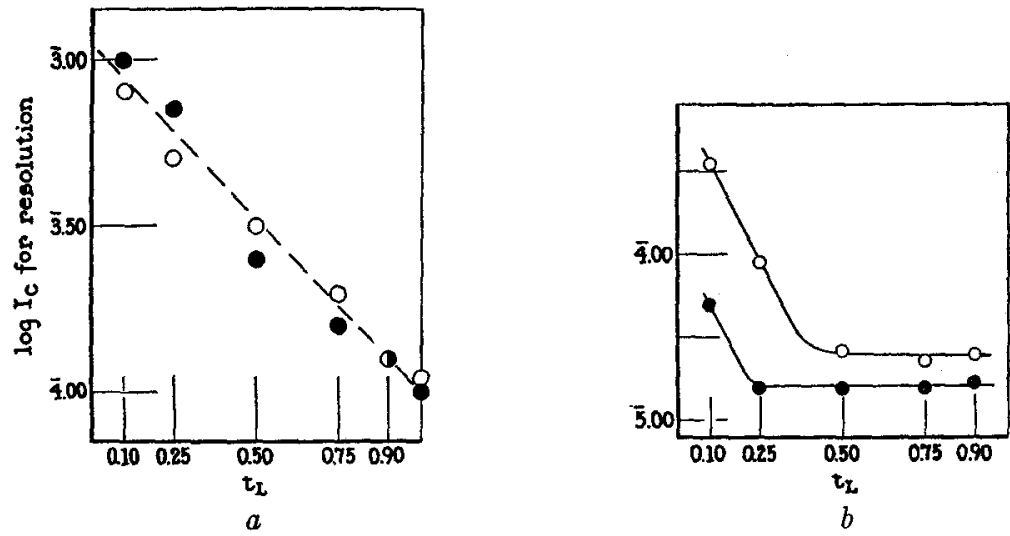

FIG. 12. Illustrating the complex dependence of visual resolution in flickered light upon flash intensity $I_{0}$ at different light-time fractions, on the flicker contours.

(a) Open circlets, 3-barred pattern (field (iii)); solid circlets, 6-barred pattern (field (iv)). The mean flux, corresponding to the Talbot brightness, is very nearly approximated at the point of resolution; thus reducing $t_{x}$ to 0.10 requires nearly ten times the flash intensity necessary with steady light.

(b) Flash intensity vs. $t_{L}$ for the resolution of the barred pattern of our "pecten" experiment; ${ }^{2,3}$ open circlets, W. J. C.; solid dots, E.W. Here, over a good range, reslution of the pattern is determined by flash intensity alone, independent of $t_{L}$.

Thus there are conditions under which resolution of a more finely subdivided field is possible with a lower flash intensity, even when the length of the subdivisions is the same. The rôle of the latter factor is easily demonstrated. ${ }^{33}$ It has been known ${ }^{24}$ that the presence of a nearby contrast border can affect the visual resolution of a given such border. The flicker data prove that phenomena of this kind, involving pronounced integration of visual functioning, can operate even when contrast as such is not perceived (i.e., below the flash intensity required for resolution of the barred pattern).

One aspect of the integrative action of an increased number of effective neural units is reflected in the associated variational indices, as we have already sug-

${ }^{33} \mathrm{~A}$ following paper deals with certain of these questions.

${ }^{34} \mathrm{Cf}$. Bartley, S. H., 1941, Vision: a study of its basis, New York, D. Van Nostrand Co., Inc., Chapter X. 
gested. In the data of Tables III and IV, $\sigma_{1} / I_{m}$ is statistically constant. The values for $\bar{\sigma}$ and for $\sigma_{\sigma}$ here differ relatively little, although those for the 4-bar field (iii) may be slightly higher. They are smaller, however, than one would be led to expect for a simple field of the same illuminated area. For a square $6^{\circ}$ field at the fovea, the values of $\bar{\sigma}$ are not different significantly, but those for $\sigma_{\sigma}$ are larger with the $10^{\circ}$ field by the average factor of 1.74 , although the fields (iii) and (iv) are half again as large, and $F_{\max }$. is decidedly higher, and we find that $\sigma_{\sigma}$ increases when the image area of a simple square is enlarged in this way. The constant $r$ is consistently larger by a little for the 7-bar field (iv) than for the 4-bar field (iii), the latter having less than two-thirds of the light-dark perimeter in the former. (Fig. 13.) This is quite striking because of the general relation previously described for simple fields between $F_{\max }$, and $r$.

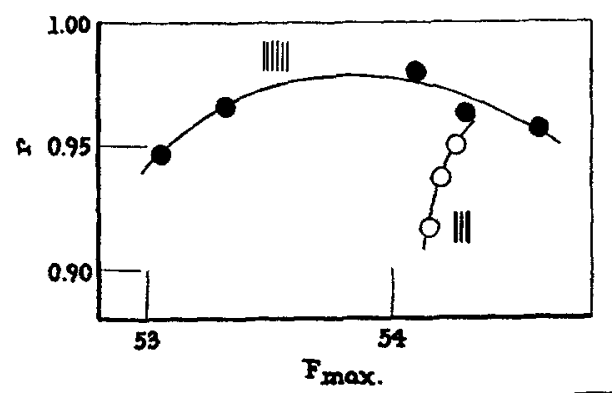

FIG. 13. The values of $r$ in the expression $\sigma_{\sigma} / \sigma \sqrt{2}=\sqrt{1-r}$ (see text), as a function of $F_{\max }$, for the series of flicker contours with fields (iii) and (iv).

We conclude, therefore, that the degree of integration involved in the recognition of visual flicker, as a function of flash frequency and intensity, is decidedly enhanced by the imposition of image forms (subdivision into stripes) such that more visual units are involved together with a reduction in the effective contribution of elements of neural action from each unit.

VI

The peculiar transposition of the $t_{L} 0.75$ and 0.90 contours in the data of section $V$ led us to inquire if we were being in some fashion misled by the occurrence of more than one intensity critical for subjective flicker, at a given $F$. It is true that under certain conditions $t_{L}=0.50$ is a critical light-time fraction, as Porter ${ }^{35}$ found for fixed illumination reflected from a spun sector disk; this result, for which an explanation has been offered, ${ }^{36}$ does not enter here. Be-

${ }^{36}$ Porter, T. C., 1898, Proc. Roy. Soc. London, 63, 347.

${ }^{36}$ 1937-38, J. Gen. Physiol., 21, 313, 463; Pieron, H., 1935, Ann. Psychol., 35, 1. 
sides, it is desirable to be able to account for the fact that the curve of $\tau^{\prime}$ as a function of $t_{L}$ exhibits a minimum at $t_{L}=0.75$ (Fig. 10).

In tests of visual acuity with grating patterns it has been found that a difference in resolvability appears when the grating stripes are at different angles to the vertical. ${ }^{37}$ For our field (iv) we find that this difference likewise appears under flickered light (Table V, Fig. 14), and also that the whole flicker contour is displaced toward slightly higher intensities when the barred field is turned

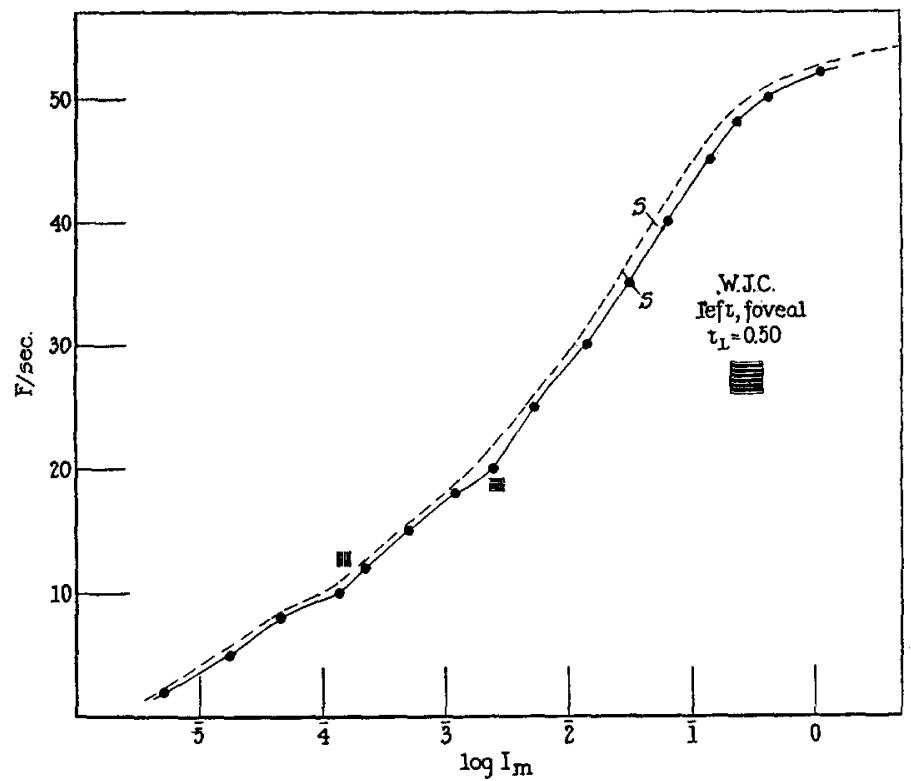

FIG. 14. Flicker contour for 6-barred field (iv) with stripes vertical (dashed line, from Fig. $8, t_{L}=0.50$ ) compared with that for the same field with the stripes rotated $90^{\circ}$, horizontal.

The point above which the horizontally barred pattern was resolved is indicated by the symbol, well above the level for the vertical bars. At $S$ the illuminated areas became smooth. See text.

with the stripes horizontal (field (v)). We doubt that gross ocular astigmatism can account for this difference, since it persists when the very slight astigmatism is suitably corrected. A more natural explanation may be found in the neural structure of the foveal region ("retinal astigmatism"). This is described as receiving rather distinct upper and lower groups of nerve fibers. ${ }^{98}$

${ }^{37}$ Shlaer, S., J. Gen. Physiol., 1937-38, 21, 165.

${ }^{38}$ Duke-Elder, W. S., Textbook of ophthalmology, St. Louis, C. V. Mosby Co., 1938, 1, 262; Polyak, S. L., The retina, Chicago, University of Chicago Press, 1941, Fig. 43. 
The suggestion is that flicker may be a little less easily perceived when groups of neighboring receptor units are simultaneously illuminated in the same manner, if their fiber connections remain together. Calculation of the $r$ constant

TABLE VI

Illustrating the relation between flash frequency and flash intensity critical for the appearance of the " $\gamma$ movement effect;" conditions as in Table $V, t_{L}=0.75$. The $\gamma$ movement thresholds are of course obtained only above flash intensities at which the illuminated bars are separately visible; see text.

\begin{tabular}{c|cc}
\hline$F$ & \multicolumn{2}{|c}{$t_{L}=0.75$} \\
per sec. & $\log I_{m}$ & $\log$ P.E.1 \\
\hline 20 & $\overline{\mathbf{5}} .7859$ & $\overline{7} .9160$ \\
25 & $\overline{4} .1309$ & $\overline{6} .4788$ \\
30 & $\overline{\mathbf{4}} .5298$ & 6.9326 \\
35 & $\overline{4} .8813$ & $\overline{\mathbf{5}} .1306$ \\
40 & $\overline{\mathbf{3}} .2127$ & $\overline{\mathbf{5}} .6238$ \\
45 & $\overline{\mathbf{3}} .6371$ & $\overline{\mathbf{5}} .8284$ \\
48 & $\overline{\mathbf{3}} .7367$ & $\overline{4} .0200$ \\
50 & $\overline{\mathbf{2}} .0269$ & $\overline{4} .3191$ \\
52 & $\overline{\mathbf{2}} .4200$ & $\overline{4} .5834$ \\
54 & $\overline{\mathbf{1}} .2709$ & $\overline{3} .5251$ \\
\hline
\end{tabular}

TABLE VII

An exceptional series of observations under the conditions specified in Table $\mathrm{V}$ for $t_{L}=$ 0.50 , involving the " $\phi$ effect;" see text.

\begin{tabular}{|c|c|c|}
\hline \multirow{2}{*}{$\frac{\begin{array}{c}F \\
\text { per sec. }\end{array}}{20}$} & \multicolumn{2}{|c|}{$\log I_{m} \quad t_{L}=0.50 \log$ P.E. } \\
\hline & $\overline{3} .4763$ & $\overline{5} .7281$ \\
\hline 25 & $\overline{3} .8308$ & $\overline{4} .2444$ \\
\hline 30 & $\overline{2} .1959$ & $\overline{4} .4441$ \\
\hline \multirow[t]{2}{*}{35} & $\overline{2} .5672$ & $\overrightarrow{4} .9065$ \\
\hline & $\overline{2} .6526$ & $\overline{4} .7402$ \\
\hline 40 & $\overline{2} .8596$ & $\overrightarrow{3} .1561$ \\
\hline \multirow[t]{2}{*}{45} & 1.2166 & $\overline{3} .5297$ \\
\hline & 1.2388 & $\overline{3} .4890$ \\
\hline \multirow[t]{2}{*}{48} & 1.6173 & $\overline{3} .7681$ \\
\hline & 1.4965 & $\overline{3} .7193$ \\
\hline \multirow[t]{2}{*}{50} & $\overline{1} .8799$ & $\overline{2} .0079$ \\
\hline & $\overline{1} .8511$ & $\overline{2} .1172$ \\
\hline 52 & 0.2804 & $\overline{2} .5742$ \\
\hline
\end{tabular}

from the flicker data shows that it is little, but significantly, lower $(0.966$ vs. 0.980 ) for the horizontal stripes. The estimated $F_{\max }$. is also a little lower. This would indicate an organizational factor, which might of itself be a function of the light-time fraction. 
We have sought diligently to see if more than one flicker end-point could be found on the intensity scale, with $F$ and $t_{L}$ fixed, but we have not found any. It is true that with the barred pattern (iv) visual disturbances of certain interesting kinds are easily recognizable at other critical intensities when the bars are almost or quite resolved, but there is no difficulty in distinguishing them from the occurrence of ficker. The most interesting of these we have regarded as an example of " $\gamma$ " apparent movement. This, most obvious at $t_{L}=0.75$, consists in a pulsatile widening and contracting of the illuminated stripes, ${ }^{39}$ at a rate lower than that of the flashes. The end-point is easily

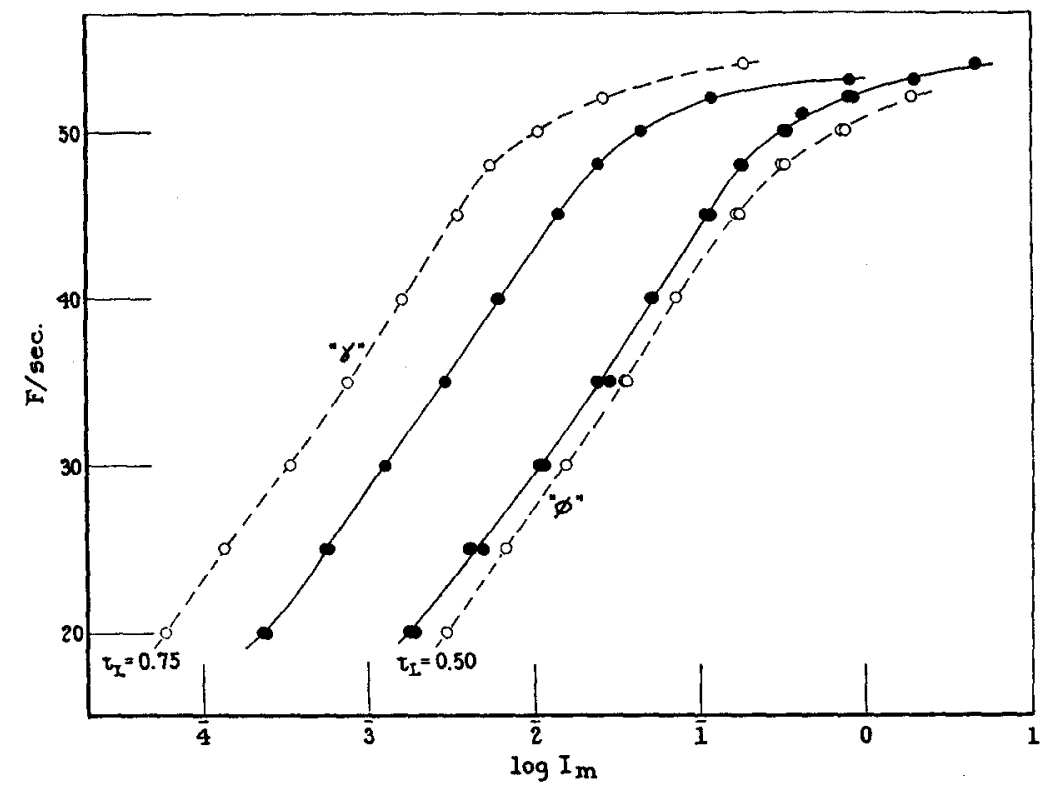

Fig. 15. Upper portions of ficker contours for $t_{L}=0.50$ and 0.75 , solid circlets, from Fig. 8, with corresponding curves for " $\gamma$ " movement and " $\varphi$ " movement. See text.

recognized. As shown in Fig. 15, the " $\gamma$ " effect provides a contour similar to that given by the flicker end-point, although at lower intensities and with a higher $F_{\max }$. (54.5 vs. 53.2 for flicker). Analysis of the curves in Fig. 15 shows that $\sigma_{\log I}^{\prime}$ is not different for these " $\gamma$ " data. It is of some interest that for the " $\gamma$ " set the relative variation $\left(\sigma_{1} / I_{m}\right)$ is lower than for flicker at $t_{L}=0.75$, by a factor of 0.835 ; and the scatter of this ratio $\left(\sigma_{\sigma}\right)$ is also lower, by the factor 0.867 , so that $r$ is not essentially different, being 0.964 vs. 0.966 .

The conclusion that the same neural units, to the same number, are con-

${ }^{39}$ Cf. Bartley, S. H., 1941, Vision: a study of its basis, New York, D. van Nostrand Co., Inc., Chapter VII. 
cerned as in the recognition of flicker, although the subjective effect used is not/the same, is supported by other series of measurements based upon what we regard as another kind of apparent movement which we may for convenience but without prejudice label the " $\varphi$ " type. ${ }^{39}$ This sort of "movement" in the subdivided visual field may be described as a kind of pulsatile pseudomovement, running from side to side of the field. We cannot be entirely certain that it is independent of eye movements. It appears at a flash intensity slightly but systematically above the unmistakable flicker end-point. In our experience with field (iv) it is best developed at $t_{L}=0.50$, and its use as an end-point gives a contour at slightly higher intensities than for flicker (Fig. 15), although again $\sigma^{\prime} \log _{I}$ is the same; $F_{\max }$. is a little lower than for flicker at the same $t_{L}(53.05 \mathrm{vs}$. 54.16); $\sigma / I_{m}$ is a little lower, $\sigma_{\sigma}$ still lower, $r$ is the same $(0.977 v .0 .980)$. It may be remarked that, just as for flicker when $t_{L}$ is varied, $\tau^{\prime}$ and $F_{\max }$. tend to change reciprocally.

In connection with the relation of (critical) flicker to other subjective phenomena we should note that a certain independence of the properties of the contours can be demonstrated as concerns brightness, ${ }^{25}$ visual resolution of the field structure, ${ }^{32}$ color ${ }^{40}$ and other phenomena. Thus, in Figs. 2, 7, 8, and 14 points along the contours are indicated at which the respective fields are no longer "speckled" or "frosted" but become "smooth" and evenly illuminated; so also in Fig. 11 these points are shown, and the color thresholds. For the " $\gamma$ " curve of Fig. 15 the lighteb dars appear smooth only above $\log I_{m}=$ $c a . \overline{1}$, and the bars are visually resolved at about $\log I_{m}=\overline{4} .0$, just as for the normal flicker end-point at $t_{L}=0.75$. The levels of occurrence of these effects, while related in interesting ways to $t_{L}$, are not associated in any manner with the presence of singularities on the $F-\log I$ curves.

To account for the relative positions of the $t_{L}$ contours with the barred fields we must turn to properties of the "edge effect." The reality of this effect is attested (1) by the systematic changes in the shapes of the curves which cannot be accounted for by changes in image area (sections III and V), and (2) by properties of the variational indices. It is also consistent with the fact that, subjectively, arrival at the critical intensity produces a more pronounced flicker along the image edges (although the whole illuminated area flickers simultaneously). To account for the non-specific nature of the shifts of $F-$ $\log I$ curves with change of temperature and of $t_{L}$ it has been proposed ${ }^{41}$ that the flash intensity critical for flicker results from the appropriate relation between the effects of flashes and of the decay of their after-effects. With the barred fields we have indication that the number of neural units concerned is large ( $\sigma_{\log I}^{\prime}$ is small). The fact that $F_{\max }$, although not very high, changes so very little with $t_{L}$ (as with field (ii) also) must be taken to mean that, although the

40 1941-42, J. Gen. Physiol., 25, 89, 293, 369; 1943-44, 27, 119.

${ }^{41}$ 1936-37, J. Gen. Physiol., 20, 393; 1937-38, 21, 313. 
mean contribution of elements of effect per neural unit is small it tends to decrease with $t_{L}$ less than might be expected. It is known that the relative expansion and contraction of an illuminated bar in $\gamma$ apparent movement is a function of $t_{L}$, and we have already pointed out that with our fields (iii), and particularly (iv), it is a maximum (in our series) at $t_{L}=0.75$. The conception then might be that the "edge" or bar effect with which $\gamma$ apparent movement is associated, and of which the latter is one expression, operates in such a way as to contribute elements of effect, from the same units, in addition to those ordinarily concerned in the recognition of flicker, and that this contribution is more marked above $t_{L}=0.50$. To the extent that, let us say, a symmetrical balance of the expanding and decaying phases of the $\gamma$ apparent movement must be achieved for effective reinforcement of the ordinary flicker effect, this could well result in a lower critical intensity for the net result with a given $F$ at the level of $t_{L}$ most efficient for the " $\gamma$ " effect. Ordinarily, with simple (rectangular) fields, above an image area of $c a .0 .73$ square degrees, the change of $\tau^{\prime}$ per unit change of $t_{L}\left(=\Delta \tau^{\prime} / \Delta t_{L}\right)$ is found to decrease steadily as the image area is increased. ${ }^{13}$ This is correlated with changes in $\sigma^{\prime}$ and in $F_{\max }$. signifying an increase in number of units concerned and a relative diminution in the number of elements per unit required to evoke flicker. In our field (ii) $\Delta \tau^{\prime}$ is considerably reduced by comparison with (i), which is consistent with the foregoing. For the striped fields (iii) and (iv) the behavior of $\tau^{\prime}$ seems to show that the number of elements of effect per neural unit required to be involved at the end-point is reduced by the "edge effect," especially at lower values of $t_{L}$. The " $\gamma$ " phenomenon appears to reverse the change of $\tau^{\prime}$ with increase of $t_{L}$ when it becomes a sufficient factor just beyond $t_{L}=0.50$. At $t_{L}=0.90$ the " $\gamma$ " effect is subjectively not so pronounced, under our conditions; a lesser contribution from it is thus to be expected, and therefore $\tau^{\prime}$ must increase in the usual way, relative to the 0.75 contour.

This interpretation is of course tentative, but some interesting tests can be based upon it.

VII

\section{SUMMARY}

Flicker contours for a square image of $3^{\circ}$ visual angle, centered $6^{\circ}$ on the temporal side of the fovea, the light sectored at a focus, are strikingly modified if the same illuminated area is arranged in four squares separated by a narrow opaque cross. The "cone" curves are made much steeper, and their abscissae of inflection $\left(\tau^{\prime}\right.$ are at higher intensities; $F_{\max }$. is not greatly changed, but alters less with change of light-time fraction in the flash cycle $\left(t_{L}\right)$. This modification is accompanied by a great enlargement of the scotopic segment of the duplex curves, consistent with the theory of the integrative relations of neural effects in the two groups of units involved. The changes are not consistent with the 
view that flicker end-points are determined by the activation of retinal cells with a fixed spatial distribution of invariable thresholds. At $t_{L}=0.50$ the $3^{\circ}$ subdivided area gives very nearly the same contour as does a square $6^{\circ} \times 6^{\circ}$, with the same total perimeter of light-dark separation; the "edge effect" thus suggested is complicated by differences in the dependence of $F_{\max }$. and $\tau^{\prime}$ upon $t_{L}$.

When an image pattern is produced by a grid of light bars separated by equally broad opaque spaces $\left(10^{\circ} \times 10^{\circ}\right.$ over-all, centered at the fovea), the photopic flicker contours are made very steep and their midpoints are situated at quite low intensities, while the "rod" contribution tends to be more completely fused with the "cone" than is found for fields not subdivided. However, instead of a progressive increase of $\tau^{\prime}$ with $t_{L}$ the curves for $t_{L}=0.75$ and 0.90 lie respectively below that for $t_{L}=0.25$ and 0.50 for a field of four broader stripes $\left(1.43^{\circ}\right)$ and both are below $t_{L}=0.25$ for a field of seven narrower stripes $\left(0.77^{\circ}\right)$. These latter changes are discussed in terms of the participation of subsidiary phenomena involving so called " $\gamma$ movement."

It is pointed out that since in these data $\sigma_{1} / I_{m}$ is for each set of conditions a statistically constant quantity with a characteristic breadth of scatter $\sigma_{\sigma}$, it is possible to calculate a "coefficient of internal correlation" $r$ which is a function of the conditions (as: image area, location, wave length of light, structure of image, light-time fraction) and which describes a property of any entire contour. The changes in $r$, as a function of the conditions of flicker excitation, reflect changes in the neural organization responsible for the liminal discrimination of flicker.

It is shown that as consequence of simple changes in the image field, three parameters, as of the probability summation, are required for the description of a simplex flicker contour - since each of these is independently modifiable as to its magnitude and in its dependence on the light-time fraction.

Subdivision of the image, with light sectored at a focus, produces in part only the changes in the flicker contour which we have earlier labelled the "pecten effect." In the latter, with light not sectored at a focus but with bar images moving across a field with inclined fixed opaque bars, the "cone" slope $(d F / d$ $\log I$ ) is sharply increased for $t_{L}>0.50$, but not below $t_{L}=0.50$, and the value of $\tau^{\prime}$ is much less than it "should be." Consequently, the change in contrast brought about by the moving contact of light/dark borders is the significant factor in the "pecten effect," not simply pulsatile interruption of the light. 